\title{
Cape Verde: Statistical Appendix
}

This Statistical Appendix for Cape Verde was prepared by a staff team of the International Monetary Fund as background documentation for the periodic consultation with the member country. It is based on the information available at the time it was completed on July 12,2006. The views expressed in this document are those of the staff team and do not necessarily reflect the views of the government of Cape Verde or the Executive Board of the IMF.

The policy of publication of staff reports and other documents by the IMF allows for the deletion of market-sensitive information.

To assist the IMF in evaluating the publication policy, reader comments are invited and may be sent by e-mail to publicationpolicy@imf.org.

Copies of this report are available to the public from

International Monetary Fund • Publication Services

700 19th Street, N.W. • Washington, D.C. 20431

Telephone: (202) 6237430 • Telefax: (202) 6237201

E-mail: publications@imf.org • Internet: http://www.imf.org

Price: $\$ 15.00$ a copy

\section{International Monetary Fund Washington, D.C.}





\section{INTERNATIONAL MONETARY FUND}

\section{CAPE VERDE}

\section{Statistical Appendix}

\section{Prepared by Mr. A. M. MacFarlan (head), Mr. N. Maehle,}

and Ms. I. Karpowicz (all AFR)

Approved by the African Department

July 12, 2006

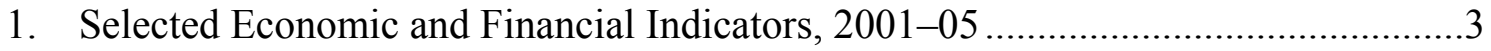

2. Selected Social and Demographic Indicators, 2001-04 .....................................4

3. Gross Domestic Product Sources and Uses of Resources, 2001-05 .........................5

4. Gross Domestic Product by Industry at Current Prices, 2001-05 ...........................

5. Gross Domestic Product by Industry at Constant 1980 Prices, 2001-05 ..................8

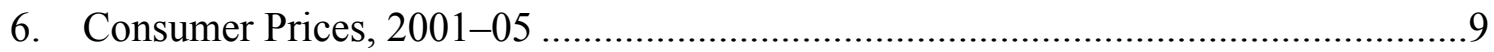

7. Fiscal Operations of the Central Government, 2001-05 ....................................10

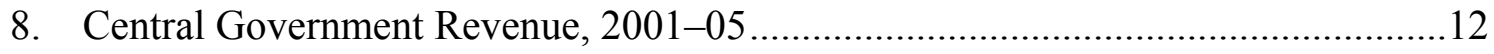

9. Selected Indicators of Central Government Revenue, 2001-05 ….......................13

10. Economic Classification of Central Government Expenditure, 2001-05 ................14

11. Public Domestic Debt of the Central Government, 2001-05 ...............................15

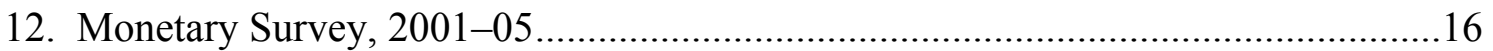

13. Summary Accounts of the Bank of Cape Verde, 2001-05 ….................................17

14. Summary Accounts of Commercial Banks, 2001-2005 ......................................18

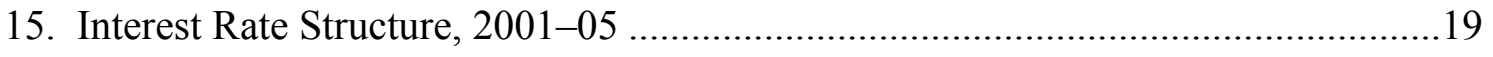

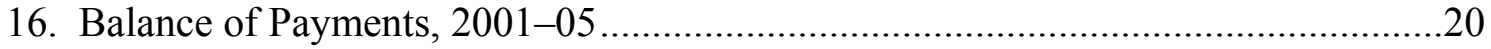

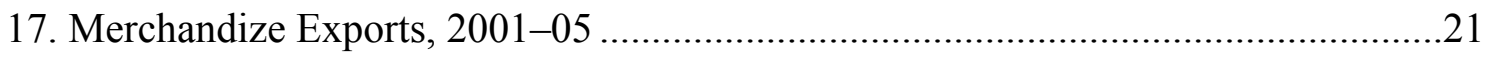

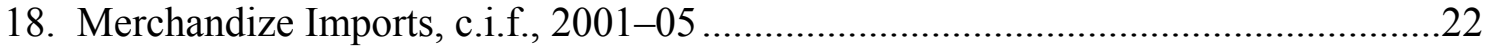




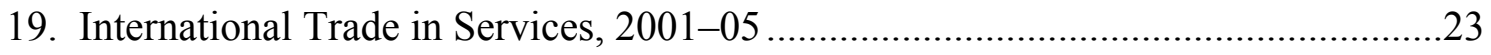

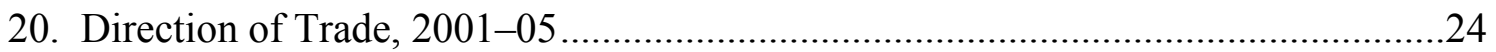

21. External Trade with CEDEAO Countries, 2001-05 .............................................25

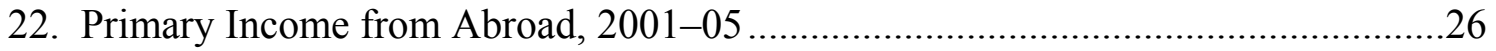

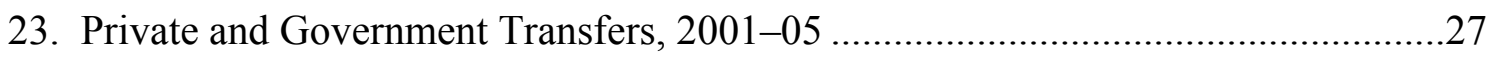

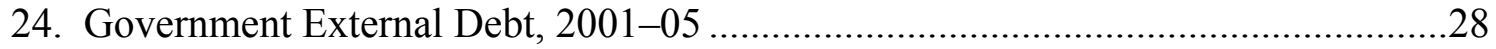

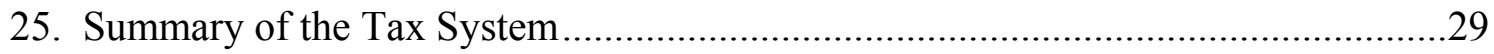


Table 1. Cape Verde: Selected Economic and Financial Indicators, 2001-05

\begin{tabular}{|c|c|c|c|c|c|}
\hline \multicolumn{6}{|l|}{ National accounts and prices } \\
\hline Constant price GDP & 6.1 & 5.3 & 4.7 & 4.4 & 5.8 \\
\hline Constant price GDP (per capita) & 4.8 & 3.4 & 2.8 & 2.5 & 3.8 \\
\hline \multicolumn{6}{|l|}{ External sector } \\
\hline Exports of goods and services & 17.9 & 14.1 & 6.1 & 5.2 & 20.5 \\
\hline Imports of goods and services & 10.8 & 15.3 & 7.5 & 6.5 & 0.5 \\
\hline Real effective exchange rate (annual average) & -2.3 & 0.5 & 1.8 & -2.9 & -2.1 \\
\hline Terms of trade (minus $=$ deterioration $)$ & 1.2 & -0.7 & 1.9 & -2.8 & -3.3 \\
\hline \multirow[t]{2}{*}{ Capital expenditure } & -9.6 & 27.8 & -18.0 & 45.8 & 3.9 \\
\hline & \multicolumn{5}{|c|}{ (Annual change in percent of beginning-of-period broad money) } \\
\hline \multicolumn{6}{|l|}{ Money and credit } \\
\hline Net foreign assets & 33.9 & 18.6 & -7.7 & 31.9 & 58.8 \\
\hline Net domestic assets & 4.9 & 13.2 & 13.2 & 5.7 & 3.4 \\
\hline Of which: net claims on the central government & 0.4 & 23.1 & 3.4 & 1.4 & -4.3 \\
\hline credit to the economy & 15.1 & 12.0 & 15.2 & 9.3 & 9.2 \\
\hline Broad money (M2) & 9.8 & 14.3 & 8.6 & 10.5 & 15.5 \\
\hline Domestic broad money (M2X) & 3.4 & 10.3 & 4.2 & 8.8 & 17.3 \\
\hline \multirow[t]{2}{*}{ Income velocity (GDP/M2) } & 1.6 & 1.5 & 1.5 & 1.4 & 1.3 \\
\hline & \multicolumn{5}{|c|}{ (Percent of GDP) } \\
\hline \multicolumn{6}{|l|}{ Government budget } \\
\hline Total revenue & 21.4 & 22.9 & 22.0 & 23.2 & 24.1 \\
\hline Total grants & 5.9 & 8.7 & 5.5 & 10.9 & 7.1 \\
\hline Total expenditure & 31.8 & 34.4 & 31.1 & 38.1 & 36.3 \\
\hline Overall balance before grants & -10.5 & -11.5 & -9.1 & -14.9 & -12.2 \\
\hline Overall balance (including grants) & -4.5 & -2.9 & -3.5 & -4.0 & -5.1 \\
\hline External financing (net) & -0.6 & -1.3 & 1.8 & 0.9 & 3.2 \\
\hline Domestic financing (net) & 2.0 & 6.5 & 2.0 & 3.8 & 1.7 \\
\hline Financing gap/ statistical discrepancy & 3.2 & -2.3 & -0.3 & -0.7 & 0.2 \\
\hline Total nominal government debt & 83.6 & 85.7 & 84.8 & 89.0 & 88.1 \\
\hline External government debt ${ }^{2}$ & 56.5 & 56.4 & 57.5 & 54.0 & 55.4 \\
\hline Domestic government debt, net of deposits ${ }^{3}$ & 27.1 & 29.2 & 27.3 & 35.0 & 32.7 \\
\hline External current account (excluding official current transfers) & -14.2 & -17.2 & -17.1 & -20.1 & -9.3 \\
\hline \multirow[t]{2}{*}{ Overall balance of payments } & 3.2 & 6.3 & -0.7 & 4.1 & 5.7 \\
\hline & \multicolumn{5}{|c|}{ (Millions of U.S. dollars, unless otherwise specified) } \\
\hline External current account (million euros, including official transfers) & -66.7 & -75.5 & -79.7 & -107.0 & -36.1 \\
\hline Gross international reserves (million euros, end of period) & 47.5 & 76.1 & 74.1 & 102.4 & 147.4 \\
\hline $\begin{array}{l}\text { Gross international reserves (months of prospective imports of } \\
\text { goods and services) }\end{array}$ & 1.3 & 1.9 & 1.7 & 2.4 & 3.0 \\
\hline External debt service (percent of exports of goods and services) & 14.2 & 12.4 & 10.5 & 11.3 & 8.8 \\
\hline
\end{tabular}

Sources: Cape Verdean authorities, and staff estimates and projections.

${ }^{1}$ Net of central government deposits. Including verified stock of domestic and external arrears.

${ }^{2}$ Measured in domestic currency. The increase in the ratio from 2004 to 2005 is due to the appreciation of the U.S. dollar in 2005.

${ }^{3}$ Excluding the claims on the offshore Trust Fund. 
Table 2. Cape Verde: Selected Social and Demographic Indicators, 2001-04

(Units as indicated)

\begin{tabular}{|c|c|c|c|c|}
\hline & 2001 & 2002 & 2003 & 2004 \\
\hline GDP per capita (current U.S. dollars) & 1192.6 & 1304.5 & 1648.5 & 1914.7 \\
\hline GDP per capita (constant 2000 U.S. dollar) & 1195.6 & 1221.4 & 1252.5 & 1290.7 \\
\hline GDP per capita, PPP (constant 2000 international dollar) & 4874.7 & 5019.3 & 5124.5 & 5263.7 \\
\hline \multicolumn{5}{|l|}{ Population } \\
\hline Total (thousand) & 461.3 & 472.4 & 483.7 & 495.2 \\
\hline Growth rate (percent a year) & 2.4 & 2.4 & 2.4 & 2.3 \\
\hline Urban population (percent of total) & 54.2 & 55.0 & 55.9 & 56.7 \\
\hline Life expectancy at birth, total (years) & .. & 69.8 & 70.1 & 70.4 \\
\hline Male & .. & 66.8 & 67.1 & 67.4 \\
\hline Female & .. & 73.0 & 73.3 & 73.6 \\
\hline Mortality rate, infant (per 1,000 live births) & .. & .. & .. & 27.0 \\
\hline Death rate, crude (per 1,000 people) & .. & 5.3 & 5.2 & 5.1 \\
\hline Birth rate, crude (per 1,000 people) & .. & 30.9 & 30.5 & 30.2 \\
\hline Fertility rate, total (births per woman) & .. & 3.8 & 3.7 & 3.6 \\
\hline \multicolumn{5}{|l|}{ Health } \\
\hline Physicians (per 1,000 people) & .. & .. & .. & 0.5 \\
\hline Hospital beds (per 1,000 people) & .. & .. & .. & .. \\
\hline \multicolumn{5}{|l|}{ Immunization rate (percent of children ages $12-23$ months) } \\
\hline DPT & 78.0 & 94.0 & 78.0 & 75.0 \\
\hline Measles & 72.0 & 85.0 & 68.0 & 69.0 \\
\hline Improved water source (percent of population with access) & .. & .. & .. & .. \\
\hline Rural (percent of rural population with access) & .. & 73.0 & .. & .. \\
\hline Urban (percent of urban population with access) & .. & 86.0 & .. & .. \\
\hline \multicolumn{5}{|l|}{ Education } \\
\hline Literacy rate, adult total (percent of people ages 15 and above) & 74.9 & 75.7 & .. & .. \\
\hline Male & 84.9 & 85.4 & .. & .. \\
\hline Female & 67.0 & 68.0 & .. & .. \\
\hline School enrollment, primary (percent net) & 96.3 & 95.1 & 94.2 & 91.8 \\
\hline Pupil-teacher ratio, primary & 28.2 & 28.8 & 27.9 & 26.9 \\
\hline Labor force, total (thousand) & 148.2 & 152.5 & 157.1 & 160.1 \\
\hline Labor force, female (percent of total labor force) & 34.2 & 33.9 & 33.4 & 33.8 \\
\hline
\end{tabular}

Source: World Development Indicators, World Bank. 
Table 3. Cape Verde: Gross Domestic Product Sources and Uses of Resources, 2001-05

(Millions of Cape Verde escudos)

\begin{tabular}{|c|c|c|c|c|c|}
\hline & 2001 & 2002 & 2003 & 2004 & 2005 \\
\hline Gross domestic product & 69,380 & 72,758 & 79,527 & 82,116 & 87,171 \\
\hline Current account & $-7,352$ & $-8,328$ & $-8,790$ & $-11,799$ & $-3,980$ \\
\hline Current account, excluding official transfers & $-9,853$ & $-12,487$ & $-13,566$ & $-16,495$ & $-8,071$ \\
\hline Resource balance & $-25,750$ & $-31,136$ & $-29,843$ & $-31,941$ & $-29,643$ \\
\hline Exports of goods and services & 13,128 & 15,209 & 11,607 & 12,214 & 14,719 \\
\hline Imports of goods and services & $-38,878$ & $-46,345$ & $-41,450$ & $-44,155$ & $-44,363$ \\
\hline Net primary income from abroad & -577 & $-1,701$ & $-1,258$ & $-1,570$ & $-2,966$ \\
\hline Government & -478 & -719 & -514 & -549 & -551 \\
\hline Nongovernment & -99 & -982 & -744 & $-1,021$ & $-2,415$ \\
\hline Gross national income & 68,804 & 71,057 & 78,268 & 80,545 & 84,205 \\
\hline Current transfers & 15,929 & 19,782 & 21,187 & 20,685 & 24,797 \\
\hline Government & 2,501 & 4,159 & 4,776 & 4,697 & 4,090 \\
\hline Nongovernment & 13,428 & 15,623 & 16,411 & 15,989 & 20,706 \\
\hline Gross national disposable income & 84,732 & 90,839 & 99,455 & 101,231 & 109,002 \\
\hline \multicolumn{6}{|l|}{ Central government } \\
\hline Current revenue & 14,542 & 16,437 & 16,972 & 18,820 & 20,976 \\
\hline Current expenditure & 14,773 & 15,693 & 17,038 & 16,924 & 18,266 \\
\hline Capital expenditure & 7,322 & 9,360 & 7,674 & 11,191 & 11,624 \\
\hline Gross capital formation & 21,979 & 26,062 & 24,672 & 30,224 & 33,019 \\
\hline Fixed capital formation & 21,985 & 26,151 & 24,735 & 30,224 & 33,019 \\
\hline Government & 4,706 & 6,702 & 5,118 & 8,023 & 10,112 \\
\hline Nongovernment & 17,279 & 19,448 & 19,617 & 22,201 & 22,907 \\
\hline Change in inventories & -6 & -89 & -63 & 0 & 0 \\
\hline Final consumption expenditures & 73,151 & 77,832 & 84,698 & 83,832 & 83,795 \\
\hline Government & 11,975 & 13,410 & 15,947 & 16,814 & 16,148 \\
\hline Households & 61,176 & 64,422 & 68,751 & 67,018 & 67,648 \\
\hline Savings & 11,582 & 13,007 & 14,757 & 17,399 & 25,207 \\
\hline Government & 1,791 & 4,184 & 4,195 & 6,044 & 6,249 \\
\hline Of which: total external grants & 4,110 & 6,319 & 4,400 & 8,946 & 6,208 \\
\hline Nongovernment & 9,791 & 8,824 & 10,562 & 11,355 & 18,958 \\
\hline
\end{tabular}

Sources: National Institute of Statistics (INE), and staff estimates. 
Table 3. Cape Verde: Gross Domestic Product Sources and Uses of Resources, 2001-05 (concluded)

(Percent of GDP)

\begin{tabular}{|c|c|c|c|c|c|}
\hline & 2001 & 2002 & 2003 & 2004 & 2005 \\
\hline Gross domestic product & 100.0 & 100.0 & 100.0 & 100.0 & 100.0 \\
\hline Current account & -10.6 & -11.4 & -11.1 & -14.4 & -4.6 \\
\hline Current account, excluding official transfers & -14.2 & -17.2 & -17.1 & -20.1 & -9.3 \\
\hline Resource balance & -37.1 & -42.8 & -37.5 & -38.9 & -34.0 \\
\hline Exports of goods and services & 18.9 & 20.9 & 14.6 & 14.9 & 16.9 \\
\hline Imports of goods and services & -56.0 & -63.7 & -52.1 & -53.8 & -50.9 \\
\hline Net primary income from abroad & -0.8 & -2.3 & -1.6 & -1.9 & -3.4 \\
\hline Government & -0.7 & -1.0 & -0.6 & -0.7 & -0.6 \\
\hline Nongovernment & -0.1 & -1.3 & -0.9 & -1.2 & -2.8 \\
\hline Gross national income & 99.2 & 97.7 & 98.4 & 98.1 & 96.6 \\
\hline Current transfers & 23.0 & 27.2 & 26.6 & 25.2 & 28.4 \\
\hline Government & 3.6 & 5.7 & 6.0 & 5.7 & 4.7 \\
\hline Nongovernment & 19.4 & 21.5 & 20.6 & 19.5 & 23.8 \\
\hline Gross national disposable income & 122.1 & 124.9 & 125.1 & 123.3 & 125.0 \\
\hline \multicolumn{6}{|l|}{ Government } \\
\hline Current revenue & 21.0 & 22.6 & 21.3 & 22.9 & 24.1 \\
\hline Current expenditure & 21.3 & 21.6 & 21.4 & 20.6 & 21.0 \\
\hline Capital expenditure & 10.6 & 12.9 & 9.6 & 13.6 & 13.3 \\
\hline Gross capital formation & 31.7 & 35.8 & 31.0 & 36.8 & 37.9 \\
\hline Fixed capital formation & 31.7 & 35.9 & 31.1 & 36.8 & 37.9 \\
\hline Government & 6.8 & 9.2 & 6.4 & 9.8 & 11.6 \\
\hline Nongovernment & 24.9 & 26.7 & 24.7 & 27.0 & 26.3 \\
\hline Change in stocks & 0.0 & -0.1 & -0.1 & 0.0 & 0.0 \\
\hline Final consumption expenditures & 105.4 & 107.0 & 106.5 & 102.1 & 96.1 \\
\hline Government & 17.3 & 18.4 & 20.1 & 20.5 & 18.5 \\
\hline Households & 88.2 & 88.5 & 86.5 & 81.6 & 77.6 \\
\hline Savings & 16.7 & 17.9 & 18.6 & 21.2 & 28.9 \\
\hline Government & 2.6 & 5.7 & 5.3 & 7.4 & 7.2 \\
\hline Of which: total external grants & 5.9 & 8.7 & 5.5 & 10.9 & 7.1 \\
\hline Nongovernment & 14.1 & 12.1 & 13.3 & 13.8 & 21.7 \\
\hline
\end{tabular}

Sources: National Institute of Statistics (INE), and staff estimates. 
Table 4. Cape Verde: Gross Domestic Product by Industry at Current Prices, 2001-05

\begin{tabular}{|c|c|c|c|c|c|}
\hline & 2001 & 2002 & 2003 & 2004 & 2005 \\
\hline & \multicolumn{5}{|c|}{ (Millions of Cape Verde escudos) } \\
\hline Agriculture, forestry, and livestock & 6,751 & 6,604 & 7,075 & 6,990 & 7,869 \\
\hline Fishing & 1,184 & 940 & 1,005 & 993 & 1,035 \\
\hline Industry and energy & 4,292 & 4,918 & 5,520 & 6,128 & 7,530 \\
\hline Construction & 5,134 & 5,888 & 6,100 & 6,358 & 9,112 \\
\hline Commerce & 11,706 & 13,554 & 14,892 & 15,515 & 18,200 \\
\hline Hotels & 1,663 & 1,583 & 1,716 & 1,644 & 2,088 \\
\hline Transport and communications & 15,803 & 14,902 & 17,124 & 17,169 & 18,621 \\
\hline Banks and insurance & 3,051 & 3,298 & 3,258 & 3,370 & 3,727 \\
\hline House renting & 3,726 & 4,028 & 4,146 & 4,223 & 5,048 \\
\hline Public service & 8,758 & 9,176 & 10,300 & 10,006 & 11,813 \\
\hline Other services & 1,513 & 1,526 & 1,682 & 1,755 & 2,078 \\
\hline Intermediary banking services & $-1,907$ & $-2,209$ & $-2,510$ & $-2,788$ & $-2,756$ \\
\hline Sum of value added ${ }^{1}$ & 61,675 & 64,208 & 70,308 & 71,365 & 84,366 \\
\hline Taxes on imports & 7,705 & 8,550 & 9,219 & 10,751 & 13,773 \\
\hline \multirow[t]{2}{*}{ Gross domestic product at market prices } & 69,380 & 72,758 & 79,527 & 82,116 & 98,139 \\
\hline & \multicolumn{5}{|c|}{ (Percent of GDP) } \\
\hline Agriculture, forestry, and livestock & 9.7 & 9.1 & 8.9 & 8.5 & 8.0 \\
\hline Fishing & 1.7 & 1.3 & 1.3 & 1.2 & 1.1 \\
\hline Industry and energy & 6.2 & 6.8 & 6.9 & 7.5 & 7.7 \\
\hline Construction & 7.4 & 8.1 & 7.7 & 7.7 & 9.3 \\
\hline Commerce & 16.9 & 18.6 & 18.7 & 18.9 & 18.5 \\
\hline Hotels & 2.4 & 2.2 & 2.2 & 2.0 & 2.1 \\
\hline Transport and communications & 22.8 & 20.5 & 21.5 & 20.9 & 19.0 \\
\hline Banks and insurance & 4.4 & 4.5 & 4.1 & 4.1 & 3.8 \\
\hline House renting & 5.4 & 5.5 & 5.2 & 5.1 & 5.1 \\
\hline Public service & 12.6 & 12.6 & 13.0 & 12.2 & 12.0 \\
\hline Other services $^{2}$ & 10.5 & 10.8 & 10.6 & 11.8 & 13.3 \\
\hline \multirow[t]{2}{*}{ Gross domestic product } & 100.0 & 100.0 & 100.0 & 100.0 & 100.0 \\
\hline & \multicolumn{5}{|c|}{ (Annual percentage change) } \\
\hline Agriculture, forestry, and livestock & -2.1 & -2.2 & 7.1 & -1.2 & 11.1 \\
\hline Fishing & -17.3 & -20.6 & 6.9 & -1.2 & 10.5 \\
\hline Industry and energy & -12.3 & 14.6 & 12.2 & 11.0 & 12.5 \\
\hline Construction & 4.0 & 14.7 & 3.6 & 4.2 & 13.3 \\
\hline Commerce & 4.1 & 15.8 & 9.9 & 4.2 & 12.3 \\
\hline Hotels & 15.0 & -4.8 & 8.4 & -4.2 & 16.7 \\
\hline Transport and communications & 24.9 & -5.7 & 14.9 & 0.3 & 10.9 \\
\hline Banks and insurance & 20.1 & 8.1 & -1.2 & 3.4 & 12.5 \\
\hline House renting & 10.6 & 8.1 & 2.9 & 1.9 & 13.5 \\
\hline Public service & 1.8 & 4.8 & 12.2 & -2.9 & 11.4 \\
\hline Other services & 4.8 & 0.9 & 10.2 & 4.3 & 13.5 \\
\hline Intermediary banking services ${ }^{1}$ & 8.0 & 15.9 & 13.6 & 11.1 & 3.4 \\
\hline Sum of value added ${ }^{2}$ & 6.9 & 4.1 & 9.5 & 1.5 & 12.4 \\
\hline Taxes on imports & 12.7 & 11.0 & 7.8 & 16.6 & 13.9 \\
\hline Gross domestic product at market prices & 7.5 & 4.9 & 9.3 & 3.3 & 12.6 \\
\hline \multicolumn{6}{|l|}{ Memorandum items: } \\
\hline Change in $\mathrm{CPI}$ & 3.7 & 1.9 & 1.2 & -1.9 & 6.2 \\
\hline Change in GDP deflator & 1.3 & -0.4 & 4.4 & -1.1 & 6.7 \\
\hline Constant price GDP growth & 6.1 & 5.3 & 4.7 & 4.4 & 5.5 \\
\hline
\end{tabular}

Sources: National Institute of Statistics, and staff estimates.

${ }^{1}$ Includes indirect taxes, net of subsidies, with the exception of taxes on imports.

${ }^{2}$ Includes "Intermediary banking services" and "Taxes and duties on imports." 
Table 5. Cape Verde: Gross Domestic Product by Industry at Constant 1980 Prices, 2001-05

\begin{tabular}{|c|c|c|c|c|c|}
\hline & 2001 & 2002 & 2003 & 2004 & 2005 \\
\hline & \multicolumn{5}{|c|}{ (Millions of Cape Verde escudos) } \\
\hline Agriculture, forestry, and livestock & 1,514 & 1,428 & 1,435 & 1,434 & 1,517 \\
\hline Fishing & 127 & 123 & 144 & 144 & 141 \\
\hline Industry and energy & 1,289 & 1,404 & 1,442 & 1,619 & 1,870 \\
\hline Construction & 1,890 & 2,184 & 2,191 & 2,311 & 2,924 \\
\hline Commerce & 3,708 & 4,215 & 4,389 & 4,625 & 5,017 \\
\hline Hotels & 671 & 660 & 811 & 787 & 939 \\
\hline Transport and communications & 4,138 & 3,951 & 4,193 & 4,255 & 4,338 \\
\hline Banks and insurance & 582 & 616 & 590 & 617 & 642 \\
\hline House renting & 1,238 & 1,325 & 1,413 & 1,457 & 1,637 \\
\hline Public service & 2,234 & 2,315 & 2,522 & 2,480 & 2,752 \\
\hline Other services & 312 & 308 & 330 & 349 & 388 \\
\hline Intermediary banking services & -401 & -457 & -514 & -578 & -537 \\
\hline Sum of value added ${ }^{2}$ & 17,303 & 18,073 & 18,947 & 19,500 & 21,628 \\
\hline Taxes on imports & 2,166 & 2,423 & 2,510 & 2,896 & 3,361 \\
\hline \multirow[t]{2}{*}{ Gross domestic product at market prices } & 19,468 & 20,496 & 21,457 & 22,397 & 24,989 \\
\hline & \multicolumn{5}{|c|}{ (Percent of GDP) } \\
\hline Agriculture, forestry, and livestock & 7.8 & 7.0 & 6.7 & 6.4 & 6.1 \\
\hline Fishing & 0.7 & 0.6 & 0.7 & 0.6 & 0.6 \\
\hline Industry and energy & 6.6 & 6.9 & 6.7 & 7.2 & 7.5 \\
\hline Construction & 9.7 & 10.7 & 10.2 & 10.3 & 11.7 \\
\hline Commerce & 19.0 & 20.6 & 20.5 & 20.7 & 20.1 \\
\hline Hotels & 3.4 & 3.2 & 3.8 & 3.5 & 3.8 \\
\hline Transport and communications & 21.3 & 19.3 & 19.5 & 19.0 & 17.4 \\
\hline Banks and insurance & 3.0 & 3.0 & 2.7 & 2.8 & 2.6 \\
\hline House renting & 6.4 & 6.5 & 6.6 & 6.5 & 6.6 \\
\hline Public service & 11.5 & 11.3 & 11.8 & 11.1 & 11.0 \\
\hline Other services ${ }^{1}$ & 10.7 & 11.1 & 10.8 & 11.9 & 12.9 \\
\hline \multirow[t]{2}{*}{ Gross domestic product at market prices } & 100.0 & 100.0 & 100.0 & 100.0 & 100.0 \\
\hline & \multicolumn{5}{|c|}{ (Percent change) } \\
\hline Agriculture, forestry, and livestock & 2.3 & -5.7 & 0.4 & 0.0 & 3.7 \\
\hline Fishing & -23.1 & -3.3 & 17.2 & 0.0 & 3.2 \\
\hline Industry and energy & -3.3 & 9.0 & 2.7 & 12.2 & 5.0 \\
\hline Construction & 4.5 & 15.6 & 0.3 & 5.5 & 10.0 \\
\hline Commerce & 11.2 & 13.6 & 4.1 & 5.4 & 4.5 \\
\hline Hotels & 29.1 & -1.5 & 22.8 & -3.0 & 9.0 \\
\hline Transport and communications & 7.6 & -4.5 & 6.1 & 1.5 & 3.5 \\
\hline Banks and insurance & 17.7 & 5.8 & -4.3 & 4.7 & 5.0 \\
\hline House renting & 5.7 & 7.0 & 6.7 & 3.1 & 6.0 \\
\hline Public service & -1.2 & 3.7 & 8.9 & -1.7 & 4.0 \\
\hline Other services & 5.5 & -1.3 & 7.3 & 5.6 & 6.0 \\
\hline Intermediary banking services & 4.8 & 14.1 & 12.4 & 12.4 & -3.5 \\
\hline Sum of value added ${ }^{2}$ & 6.0 & 4.5 & 4.8 & 2.9 & 5.5 \\
\hline Taxes on imports & 7.3 & 11.9 & 3.6 & 15.4 & 5.5 \\
\hline Gross domestic product at market prices & 6.1 & 5.3 & 4.7 & 4.4 & 5.5 \\
\hline
\end{tabular}

Sources: National Institute of Statistics, and staff estimates.

' Includes "Intermediary banking services" and "Taxes and duties on imports."

${ }^{2}$ Includes indirect taxes, net of subsidies, with the exception of taxes on imports. 
Table 6. Cape Verde: Consumer Prices, 2001-05

\begin{tabular}{|c|c|c|c|c|c|}
\hline & 2001 & 2002 & 2003 & 2004 & 2005 \\
\hline & \multicolumn{5}{|c|}{$($ Index: $1989=100)$} \\
\hline January & 173.8 & 183.0 & 189.5 & 184.7 & 183.8 \\
\hline February & 174.1 & 182.8 & 190.8 & 183.9 & 183.0 \\
\hline March & 178.9 & 183.8 & 189.9 & 184.6 & 183.0 \\
\hline April & 181.9 & 184.1 & 188.9 & 183.8 & 183.1 \\
\hline May & 182.6 & 186.0 & 188.6 & 185.1 & 182.5 \\
\hline June & 182.1 & 185.6 & 188.9 & 184.2 & 183.9 \\
\hline July & 184.3 & 185.9 & 189.4 & 185.1 & 186.1 \\
\hline August & 188.7 & 187.3 & 189.1 & 186.1 & 189.7 \\
\hline September & 189.7 & 188.9 & 188.9 & 186.0 & 189.9 \\
\hline October & 187.5 & 188.3 & 186.4 & 185.3 & 187.7 \\
\hline November & 185.0 & 189.3 & 185.4 & 185.0 & 187.2 \\
\hline December & 185.0 & 190.5 & 186.2 & 185.6 & 188.8 \\
\hline \multirow[t]{2}{*}{ Average } & 182.8 & 186.3 & 188.5 & 184.9 & 185.7 \\
\hline & \multicolumn{5}{|c|}{ (Monthly percentage changes) } \\
\hline January & -1.8 & -1.1 & -0.5 & -0.8 & -1.0 \\
\hline February & 0.2 & -0.1 & 0.7 & -0.4 & -0.5 \\
\hline March & 2.8 & 0.5 & -0.5 & 0.4 & 0.0 \\
\hline April & 1.7 & 0.2 & -0.5 & -0.4 & 0.0 \\
\hline May & 0.4 & 1.0 & -0.2 & 0.7 & -0.3 \\
\hline June & -0.2 & -0.2 & 0.1 & -0.5 & 0.8 \\
\hline July & 1.2 & 0.2 & 0.3 & 0.5 & 1.2 \\
\hline August & 2.4 & 0.7 & -0.2 & 0.6 & 2.0 \\
\hline September & 0.5 & 0.9 & -0.1 & -0.1 & 0.1 \\
\hline October & -1.1 & -0.3 & -1.3 & -0.4 & -1.2 \\
\hline November & -1.3 & 0.5 & -0.6 & -0.2 & -0.3 \\
\hline December & 0.0 & 0.7 & 0.4 & 0.3 & 0.9 \\
\hline \multirow[t]{2}{*}{ Average } & 0.4 & 0.2 & -0.2 & 0.0 & 0.1 \\
\hline & \multicolumn{5}{|c|}{ (Annual percentage changes) } \\
\hline January & -1.2 & 5.3 & 3.6 & -2.6 & -0.5 \\
\hline February & -0.6 & 5.0 & 4.4 & -3.6 & -0.5 \\
\hline March & 3.3 & 2.7 & 3.4 & -2.8 & -0.9 \\
\hline April & 4.1 & 1.2 & 2.6 & -2.7 & -0.4 \\
\hline May & 4.3 & 1.9 & 1.4 & -1.9 & -1.4 \\
\hline June & 4.0 & 1.9 & 1.8 & -2.5 & -0.1 \\
\hline July & 5.2 & 0.9 & 1.9 & -2.3 & 0.6 \\
\hline August & 4.5 & -0.7 & 0.9 & -1.5 & 1.9 \\
\hline September & 5.8 & -0.4 & 0.0 & -1.5 & 2.1 \\
\hline October & 5.3 & 0.4 & -1.0 & -0.6 & 1.3 \\
\hline November & 4.6 & 2.3 & -2.1 & -0.2 & 1.2 \\
\hline December & 4.6 & 3.0 & -2.3 & -0.3 & 1.7 \\
\hline Average & 3.7 & 1.9 & 1.2 & -1.9 & 0.4 \\
\hline
\end{tabular}

Sources: National Institute of Statistics (INE). 
Table 7. Cape Verde: Fiscal Operations of the Central Government, 2001-05

\begin{tabular}{|c|c|c|c|c|c|}
\hline & 2001 & 2002 & 2003 & 2004 & 2005 \\
\hline & \multicolumn{5}{|c|}{ (Billions of Cape Verde escudos) } \\
\hline Revenue, grants, and net lending & 18.9 & 23.0 & 21.9 & 28.0 & 27.2 \\
\hline Domestic revenue & 14.8 & 16.7 & 17.5 & 19.0 & 21.0 \\
\hline Tax revenue & 13.0 & 14.7 & 15.5 & 16.6 & 18.6 \\
\hline Income and profit taxes & 4.8 & 5.1 & 5.3 & 5.4 & 5.8 \\
\hline Consumption taxes & 2.1 & 2.5 & 2.5 & 6.3 & 7.4 \\
\hline International trade taxes & 5.3 & 6.1 & 6.8 & 3.9 & 4.2 \\
\hline Other taxes & 0.8 & 0.9 & 0.9 & 1.0 & 1.2 \\
\hline Nontax revenue & 1.5 & 1.8 & 1.5 & 1.9 & 2.2 \\
\hline Fees and penalties & 0.3 & 0.3 & 0.4 & 0.5 & 1.2 \\
\hline Other nontax revenues & 0.6 & 0.6 & 0.9 & 1.4 & 1.0 \\
\hline Profit transfers & 0.6 & 0.8 & 0.3 & 0.0 & 0.0 \\
\hline Domestic capital participation & 0.0 & 0.0 & 0.0 & 0.2 & 0.2 \\
\hline External grants & 4.1 & 6.3 & 4.4 & 8.9 & 6.2 \\
\hline Capital grants & 4.1 & 5.2 & 3.6 & 7.7 & 5.7 \\
\hline Budget support & 0.0 & 1.2 & 0.8 & 1.2 & 0.6 \\
\hline Net lending & 0.3 & 0.2 & 0.0 & 0.1 & 0.3 \\
\hline Total expenditure & 22.1 & 25.1 & 24.7 & 31.3 & 31.6 \\
\hline Recurrent expenditure & 14.8 & 15.5 & 16.4 & 16.9 & 18.3 \\
\hline Primary current expenditure & 13.4 & 13.3 & 14.4 & 14.9 & 16.3 \\
\hline Wages and salaries & 6.6 & 7.1 & 9.4 & 9.8 & 10.7 \\
\hline Goods and services & 0.6 & 0.6 & 1.3 & 1.0 & 1.2 \\
\hline Transfers and subsidies ${ }^{1}$ & 5.7 & 4.9 & 3.0 & 3.0 & 3.1 \\
\hline Other expenditures & 0.5 & 0.7 & 0.8 & 1.1 & 1.3 \\
\hline Domestic interest payments & 0.9 & 1.4 & 1.5 & 1.5 & 1.4 \\
\hline External interest payments & 0.5 & 0.7 & 0.5 & 0.5 & 0.5 \\
\hline Extraordinary expenditures & 0.0 & 0.2 & 0.6 & 0.0 & 0.0 \\
\hline Social emergency measures & 0.0 & 0.0 & 0.0 & 0.0 & 0.0 \\
\hline Restructuring costs & 0.0 & 0.2 & 0.6 & 0.0 & 0.0 \\
\hline Capital expenditure & 7.3 & 9.4 & 7.7 & 11.2 & 11.6 \\
\hline Foreign financed & 6.2 & 8.6 & 6.9 & 10.3 & 9.8 \\
\hline Domestically financed & 1.1 & 0.7 & 0.8 & 0.9 & 1.9 \\
\hline Overall balance, including grants ${ }^{2}$ & -3.2 & -2.1 & -2.8 & -3.3 & -4.4 \\
\hline Financing & 3.2 & 2.1 & 2.8 & 3.3 & 4.4 \\
\hline Foreign (net) & -0.4 & -1.0 & 1.4 & 0.7 & 2.8 \\
\hline Total drawings & 1.9 & 2.8 & 3.5 & 2.9 & 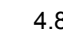 \\
\hline Balance of payments, budget & -1.2 & 0.1 & 1.3 & 0.3 & 1.3 \\
\hline Bridge loans & 0.0 & 0.5 & 0.0 & 0.0 & 0.0 \\
\hline Credit facility (FAC) & 0.6 & 0.6 & 0.6 & 0.5 & 0.0 \\
\hline Other & -1.8 & -1.0 & 0.8 & -0.2 & 0.0 \\
\hline Project loans & 3.1 & 2.7 & 2.2 & 2.6 & 3.5 \\
\hline Amortization & -2.4 & -2.2 & -2.1 & -2.4 & -2.0 \\
\hline Bridge loans and Portuguese credit facility & -1.8 & -1.1 & -1.1 & -1.1 & -0.1 \\
\hline Other & -0.7 & -1.1 & -1.0 & -1.3 & -1.9 \\
\hline Change in arrears & 1.4 & 0.0 & 0.0 & 0.0 & 0.0 \\
\hline Domestic (net) & 1.4 & 4.7 & 1.6 & 3.1 & 1.5 \\
\hline Banking system $^{3}$ & 0.1 & 2.7 & 0.9 & 0.6 & 0.6 \\
\hline Nonbanks & 1.3 & 2.0 & 0.4 & 1.0 & 0.6 \\
\hline Domestic arrears & 0.0 & 0.0 & 0.0 & 0.0 & 0.0 \\
\hline Net errors and omissions & 2.2 & -1.6 & -0.3 & -0.6 & 0.2 \\
\hline Financing gap & 0.0 & 0.0 & 0.0 & 0.0 & 0.0 \\
\hline \multicolumn{6}{|l|}{ Memorandum items: } \\
\hline Overall balance, excluding grants ${ }^{4}$ & -7.3 & -8.4 & -7.2 & -12.2 & -10.6 \\
\hline Primary current balance ${ }^{5}$ & 1.5 & 3.3 & 3.1 & 4.2 & 4.6 \\
\hline Primary balance ${ }^{6}$ & -5.8 & -6.2 & -5.2 & -10.2 & -8.7 \\
\hline Domestic balance $^{7}$ & -0.3 & 1.2 & 0.8 & -0.9 & -0.2 \\
\hline
\end{tabular}

Sources: Ministry of Finance and Public Administration, Bank of Cape Verde, and staff estimates. 
Table 7. Cape Verde: Fiscal Operations of the Central Government, 2001-05 (concluded)

\begin{tabular}{|c|c|c|c|c|c|}
\hline & 2001 & 2002 & 2003 & 2004 & 2005 \\
\hline & \multicolumn{5}{|c|}{ (Percent of GDP) } \\
\hline Revenue, grants, and net lending & 27.3 & 31.6 & 27.6 & 34.1 & 31.2 \\
\hline Domestic revenue & 21.4 & 22.9 & 22.0 & 23.2 & 24.1 \\
\hline Tax revenue & 18.7 & 20.2 & 19.4 & 20.3 & 21.3 \\
\hline Income and profit taxes & 6.9 & 7.1 & 6.7 & 6.6 & 6.7 \\
\hline Consumption taxes & 3.0 & 3.4 & 3.1 & 7.7 & 8.5 \\
\hline International trade taxes & 7.7 & 8.4 & 8.5 & 4.8 & 4.9 \\
\hline Other taxes & 1.1 & 1.3 & 1.1 & 1.2 & 1.3 \\
\hline Nontax revenue & 2.2 & 2.4 & 1.9 & 2.4 & 2.5 \\
\hline Fees and penalties & 0.5 & 0.4 & 0.4 & 0.6 & 1.4 \\
\hline Other nontax revenues & 0.8 & 0.8 & 1.1 & 1.7 & 1.1 \\
\hline Profit transfers & 0.9 & 1.1 & 0.4 & 0.0 & 0.0 \\
\hline Domestic capital participation & 0.0 & 0.0 & 0.0 & 0.3 & 0.2 \\
\hline External grants & 5.9 & 8.7 & 5.5 & 10.9 & 7.1 \\
\hline Capital grants & 5.9 & 7.1 & 4.6 & 9.4 & 6.5 \\
\hline Budget support & 0.0 & 1.6 & 1.0 & 1.5 & 0.6 \\
\hline Net lending & 0.4 & 0.3 & 0.0 & 0.1 & 0.3 \\
\hline Total expenditure & 31.8 & 34.4 & 31.1 & 38.1 & 36.3 \\
\hline Recurrent expenditure & 21.3 & 21.3 & 20.6 & 20.6 & 21.0 \\
\hline Primary current expenditure & 19.3 & 18.3 & 18.1 & 18.1 & 18.7 \\
\hline Wages and salaries & 9.5 & 9.7 & 11.8 & 11.9 & 12.3 \\
\hline Goods and services & 0.8 & 0.8 & 1.7 & 1.2 & 1.3 \\
\hline Transfers and subsidies ${ }^{1}$ & 8.2 & 6.7 & 3.7 & 3.7 & 3.6 \\
\hline Other expenditures & 0.7 & 1.0 & 1.0 & 1.3 & 1.5 \\
\hline Domestic interest payments & 1.4 & 2.0 & 1.9 & 1.8 & 1.6 \\
\hline External interest payments & 0.7 & 1.0 & 0.6 & 0.7 & 0.6 \\
\hline Extraordinary expenditures & 0.0 & 0.3 & 0.8 & 0.0 & 0.0 \\
\hline Social emergency measures & 0.0 & 0.0 & 0.0 & 0.0 & 0.0 \\
\hline Restructuring costs & 0.0 & 0.3 & 0.8 & 0.0 & 0.0 \\
\hline Capital expenditure & 10.6 & 12.9 & 9.6 & 13.6 & 13.3 \\
\hline Foreign financed & 8.9 & 11.9 & 8.7 & 12.6 & 11.2 \\
\hline Domestically financed & 1.6 & 1.0 & 1.0 & 1.1 & 2.1 \\
\hline Overall balance, including grants ${ }^{2}$ & -4.5 & -2.9 & -3.5 & -4.0 & -5.1 \\
\hline Financing & 4.5 & 2.9 & 3.5 & 4.0 & 5.1 \\
\hline Foreign (net) & -0.6 & -1.3 & 1.8 & 0.9 & 3.2 \\
\hline Total drawings & 2.8 & 3.9 & 4.5 & 3.6 & 5.5 \\
\hline Balance of payments, budget & -1.7 & 0.1 & 1.7 & 0.4 & 1.5 \\
\hline Project loans & 4.5 & 3.7 & 2.8 & 3.2 & 4.0 \\
\hline Amortization & -3.5 & -3.0 & -2.6 & -2.9 & -2.3 \\
\hline Bridge loans and Portuguese credit facility & -2.5 & -1.5 & -1.4 & -1.3 & -0.1 \\
\hline Other & -1.0 & -1.5 & -1.3 & -1.6 & -2.2 \\
\hline Change in arrears & 2.0 & 0.0 & 0.0 & 0.0 & 0.0 \\
\hline Domestic (net) & 2.0 & 6.5 & 2.0 & 3.8 & 1.7 \\
\hline Banking system & 0.1 & 3.7 & 1.2 & 0.8 & 0.7 \\
\hline Nonbanks & 1.9 & 2.8 & 0.5 & 1.2 & 0.7 \\
\hline Domestic arrears & 0.0 & 0.0 & 0.0 & 0.0 & 0.0 \\
\hline Net errors and omissions & 3.2 & -2.3 & -0.3 & -0.7 & 0.2 \\
\hline Financing gap & 0.0 & 0.0 & 0.0 & 0.0 & 0.0 \\
\hline \multicolumn{6}{|l|}{ Memorandum items: } \\
\hline Overall balance, excluding grants ${ }^{4}$ & -10.5 & -11.5 & -9.1 & -14.9 & -12.2 \\
\hline Primary current balance ${ }^{5}$ & 2.1 & 4.6 & 3.9 & 5.1 & 5.3 \\
\hline Primary balance ${ }^{6}$ & -8.4 & -8.6 & -6.5 & -12.4 & -10.0 \\
\hline Domestic balance $^{7}$ & -0.4 & 1.6 & 1.0 & -1.1 & -0.2 \\
\hline
\end{tabular}

Sources: Ministry of Finance and Public Administration, Bank of Cape Verde, and staff estimates.

${ }^{1}$ Includes student scholarships.

${ }^{2}$ Overall balance, incl. grants $=$ revenue, incl. grants - total expenditure .

${ }^{3}$ Due to TCMF operations, bank financing in 1999 and 2000 does not match change in banking systems' net credit to central government.

${ }^{4}$ Overall balance, excl. grants $=$ revenue, excl. grants - total expenditure.

${ }^{5}$ Primary current balance $=$ domestic revenue - primary current expenditure

${ }^{6}$ Primary balance $=$ domestic revenue - total expenditure + domestic and external interest payments

' Domestic balance $=$ domestic revenue - recurrent expenditure + external interest payments - extraordinary expenditures -

domestically financed capital expenditure. 
Table 8. Cape Verde: Central Government Revenue, 2001-05

(Millions of Cape Verde escudos, unless otherwise indicated)

\begin{tabular}{|c|c|c|c|c|c|}
\hline & 2001 & 2002 & 2003 & 2004 & 2005 \\
\hline Tax revenue & 12,988 & 14,678 & 15,457 & 16,638 & 18,601 \\
\hline Direct taxes & 4,788 & 5,132 & 5,304 & 5,395 & 5,822 \\
\hline Unified tax on income and profits & 4,788 & 5,132 & 5,304 & 5,395 & 5,822 \\
\hline Other direct taxes & 0 & 0 & 0 & 0 & 0 \\
\hline Indirect taxes & 8,199 & 9,546 & 10,153 & 11,244 & 12,779 \\
\hline Taxes on international trade & 5,341 & 6,127 & 6,755 & 3,914 & 4,231 \\
\hline Import duties & 3,705 & 4,042 & 4,330 & 3,853 & 4,231 \\
\hline Customs services fee & 1,624 & 2,072 & 2,412 & 60 & 0 \\
\hline Other taxes & 11 & 13 & 13 & 2 & 0 \\
\hline Consumption taxes & 2,099 & 2,470 & 2,500 & 6,347 & 7,390 \\
\hline Domestic goods and services & 63 & 108 & 100 & 8 & 0 \\
\hline International goods and services ${ }^{1}$ & 1,712 & 1,798 & 1,934 & 747 & 838 \\
\hline Tax on petroleum products & 122 & 315 & 218 & 0 & 0 \\
\hline Liquor tax & 105 & 125 & 126 & 0 & 0 \\
\hline Tourism tax & 98 & 124 & 122 & 0 & 0 \\
\hline Municipal taxes ${ }^{2}$ & 68 & 113 & 83 & 115 & 192 \\
\hline Ecological tax & 68 & 113 & 83 & 115 & 192 \\
\hline Other municipal taxes & 0 & 0 & 0 & 0 & 0 \\
\hline Other indirect taxes & 691 & 836 & 815 & 868 & 966 \\
\hline Stamp tax & 591 & 723 & 678 & 733 & 810 \\
\hline Other & 100 & 114 & 137 & 135 & 156 \\
\hline Nontax revenue & 1,543 & 1,759 & 1,514 & 1,933 & 2,205 \\
\hline Fees and penalties & 339 & 320 & 355 & 509 & 1,220 \\
\hline Property income & 645 & 832 & 294 & 30 & 34 \\
\hline Transfers & 384 & 294 & 436 & 542 & 597 \\
\hline Sales of fixed assets and services & 153 & 154 & 149 & 160 & 168 \\
\hline Autonomous revenues ${ }^{3}$ & 0 & 0 & 0 & 0 & 0 \\
\hline Other revenues & 22 & 158 & 281 & 693 & 186 \\
\hline Total budgetary revenue ${ }^{4}$ & 14,834 & 16,658 & 17,509 & 19,026 & 20,976 \\
\hline Annual percent change & 12.1 & 12.3 & 5.1 & 8.7 & 10.2 \\
\hline
\end{tabular}

Source: Ministry of Finance and Public Administration.

${ }^{1}$ Collected by the customs department on imports.

${ }^{2}$ In 1998, municipal taxes were collected by the central government and directly passed on to the municipalities.

${ }^{3}$ These are called contas de ordem, which are revenue as budgeted from the direct provision of services by government agencies, offset by the same amounts of current expenditure for each of these agencies.

${ }^{4}$ Excludes revenue from domestic capital participation and net lending. 
Table 9. Cape Verde: Selected Indicators of Central Government Revenue, 2001-05

\begin{tabular}{|c|c|c|c|c|c|}
\hline & 2001 & 2002 & 2003 & 2004 & 2005 \\
\hline & \multicolumn{5}{|c|}{ (Millions of Cape Verde escudos) } \\
\hline Tax revenue & 12,988 & 14,678 & 15,457 & 16,638 & 18,601 \\
\hline Of which: taxes on income and profits & 4,788 & 5,132 & 5,304 & 5,395 & 5,822 \\
\hline taxes on international trade & 5,341 & 6,127 & 6,755 & 3,914 & 4,231 \\
\hline consumption taxes & 2,099 & 2,470 & 2,500 & 6,347 & 7,390 \\
\hline Nontax revenue & 1,543 & 1,759 & 1,514 & 1,933 & 2,205 \\
\hline Of which: property income & 645 & 832 & 294 & 30 & 34 \\
\hline transfers & 384 & 294 & 436 & 542 & 597 \\
\hline \multirow[t]{2}{*}{ sale of fixed assets } & 153 & 154 & 149 & 160 & 168 \\
\hline & \multicolumn{5}{|c|}{ (Percent of budgetary revenue) } \\
\hline Tax revenue & 87.6 & 88.1 & 88.3 & 87.5 & 88.7 \\
\hline Of which: taxes on income and profits & 32.3 & 30.8 & 30.3 & 28.4 & 27.8 \\
\hline taxes on international trade & 36.0 & 36.8 & 38.6 & 20.6 & 20.2 \\
\hline consumption taxes & 14.2 & 14.8 & 14.3 & 33.4 & 35.2 \\
\hline Nontax revenue & 10.4 & 10.6 & 8.6 & 10.2 & 10.5 \\
\hline Of which: property income & 4.4 & 5.0 & 1.7 & 0.2 & 0.2 \\
\hline transfers & 2.6 & 1.8 & 2.5 & 2.8 & 2.8 \\
\hline \multirow[t]{2}{*}{ sale of fixed assets } & 1.0 & 0.9 & 0.8 & 0.8 & 0.8 \\
\hline & \multicolumn{5}{|c|}{ (Annual percentage change) } \\
\hline Tax revenue & 10.4 & 13.0 & 5.3 & 7.6 & 11.8 \\
\hline Of which: taxes on income and profits & 22.5 & 7.2 & 3.4 & 1.7 & 7.9 \\
\hline taxes on international trade & 11.1 & 14.7 & 10.3 & -42.1 & 8.1 \\
\hline consumption taxes & 11.1 & 17.7 & 1.2 & 153.9 & 16.4 \\
\hline Nontax revenue & 4.4 & 14.0 & -13.9 & 27.7 & 14.1 \\
\hline Of which: property income & $6,120.6$ & 28.9 & -64.7 & -89.8 & 12.9 \\
\hline transfers & -34.6 & -23.4 & 48.1 & 24.2 & 10.2 \\
\hline \multirow[t]{2}{*}{ sale of fixed assets } & -23.8 & 1.0 & -3.7 & 7.5 & 5.4 \\
\hline & \multicolumn{5}{|c|}{ (Percent of GDP) } \\
\hline Tax revenue & 18.7 & 20.2 & 19.4 & 20.3 & 21.3 \\
\hline Of which: taxes on income and profits & 6.9 & 7.1 & 6.7 & 6.6 & 6.7 \\
\hline taxes on international trade & 7.7 & 8.4 & 8.5 & 4.8 & 4.9 \\
\hline consumption taxes & 3.0 & 3.4 & 3.1 & 7.7 & 8.5 \\
\hline Nontax revenue & 2.2 & 2.4 & 1.9 & 2.4 & 2.5 \\
\hline Of which: property income & 0.9 & 1.1 & 0.4 & 0.0 & 0.0 \\
\hline transfers & 0.6 & 0.4 & 0.5 & 0.7 & 0.7 \\
\hline sale of fixed assets & 0.2 & 0.2 & 0.2 & 0.2 & 0.2 \\
\hline
\end{tabular}

Sources: Ministry of Finance and Public Administration, and staff estimates. 
Table 10. Cape Verde: Economic Classification of Central Government Expenditure, 2001-05

\begin{tabular}{|c|c|c|c|c|c|}
\hline & 2001 & 2002 & 2003 & 2004 & 2005 \\
\hline & \multicolumn{5}{|c|}{ (Millions of Cape Verde escudos) } \\
\hline Total expenditure & 22,095 & 25,053 & 24,712 & 31,251 & 31,615 \\
\hline Recurrent expenditure & 14,773 & 15,468 & 16,408 & 16,924 & 18,266 \\
\hline Wages and salaries & 6,577 & 7,092 & 9,361 & 9,805 & 10,733 \\
\hline Goods and services & 566 & 604 & 1,319 & 982 & 1,157 \\
\hline Interest on public debt & 1,415 & 2,157 & 1,995 & 2,056 & 1,930 \\
\hline Domestic $^{1}$ & 937 & 1,437 & 1,480 & 1,506 & 1,384 \\
\hline External $^{2}$ & 478 & 719 & 514 & 550 & 546 \\
\hline Subsidies and transfers & 5,717 & 4,889 & 2,969 & 2,998 & 3,149 \\
\hline Public enterprises & 0 & 0 & 0 & 0 & 0 \\
\hline Public sector & 4,041 & 4,199 & 2,334 & 2,478 & 2,616 \\
\hline Other entities & 1,677 & 690 & 634 & 519 & 533 \\
\hline Other current expenditure & 498 & 726 & 765 & 1,084 & 1,298 \\
\hline Capital expenditure & 7,322 & 9,360 & 7,674 & 11,191 & 11,624 \\
\hline Foreign financing & 6,189 & 8,638 & 6,912 & 10,311 & 9,755 \\
\hline Grants & 4,110 & 6,319 & 4,400 & 8,946 & 6,208 \\
\hline Loans & 2,080 & 2,319 & 2,512 & 1,365 & 3,547 \\
\hline Domestic resources & 1,132 & 723 & 762 & 880 & 1,869 \\
\hline \multirow[t]{2}{*}{ Other expenditures ${ }^{3}$} & 0 & 225 & 630 & 3,137 & 1,725 \\
\hline & \multicolumn{5}{|c|}{ (Percent of recurrent expenditure) } \\
\hline Recurrent expenditure & 100.0 & 100.0 & 100.0 & 100.0 & 100.0 \\
\hline Wages and salaries & 44.5 & 45.9 & 57.0 & 57.9 & 58.8 \\
\hline Goods and services & 3.8 & 3.9 & 8.0 & 5.8 & 6.3 \\
\hline Interest on public debt & 9.6 & 13.9 & 12.2 & 12.1 & 10.6 \\
\hline Domestic $^{1}$ & 6.3 & 9.3 & 9.0 & 8.9 & 7.6 \\
\hline External $^{2}$ & 3.2 & 4.7 & 3.1 & 3.2 & 3.0 \\
\hline Subsidies and transfers & 38.7 & 31.6 & 18.1 & 17.7 & 17.2 \\
\hline Public enterprises & 0.0 & 0.0 & 0.0 & 0.0 & 0.0 \\
\hline Public sector & 27.4 & 27.1 & 14.2 & 14.6 & 14.3 \\
\hline Other entities & 11.3 & 4.5 & 3.9 & 3.1 & 2.9 \\
\hline \multirow[t]{2}{*}{ Other current expenditure } & 3.4 & 4.7 & 4.7 & 6.4 & 7.1 \\
\hline & \multicolumn{5}{|c|}{ (Percent of GDP) } \\
\hline Recurrent expenditure & 21.3 & 21.3 & 20.6 & 20.6 & 21.0 \\
\hline Wages and salaries & 9.5 & 9.7 & 11.8 & 11.9 & 12.3 \\
\hline Goods and services & 0.8 & 0.8 & 1.7 & 1.2 & 1.3 \\
\hline Interest on public debt & 2.0 & 3.0 & 2.5 & 2.5 & 2.2 \\
\hline Domestic $^{1}$ & 1.4 & 2.0 & 1.9 & 1.8 & 1.6 \\
\hline External $^{2}$ & 0.7 & 1.0 & 0.6 & 0.7 & 0.6 \\
\hline Subsidies and transfers & 8.2 & 6.7 & 3.7 & 3.7 & 3.6 \\
\hline Public enterprises & 0.0 & 0.0 & 0.0 & 0.0 & 0.0 \\
\hline Public sector & 5.8 & 5.8 & 2.9 & 3.0 & 3.0 \\
\hline Other entities & 2.4 & 0.9 & 0.8 & 0.6 & 0.6 \\
\hline Other current expenditure & 0.7 & 1.0 & 1.0 & 1.3 & 1.5 \\
\hline
\end{tabular}

Sources: Ministry of Finance and Public Administration, and staff estimates.

${ }^{1}$ Including the debt service to the central bank and the Instituto Nacional de Previdencia Social (INPS).

¿ Including central government and public enterprises' debt guaranteed by the central government.

${ }^{3}$ Includes arrears clearance, restructuring costs, and unspecified expenditures. 
Table 11. Cape Verde: Public Domestic Debt of the Central Government, 2001-05

(Millions of Cape Verde escudos)

\begin{tabular}{|c|c|c|c|c|c|}
\hline & 2001 & 2002 & 2003 & 2004 & 2005 \\
\hline Stock of domestic debt, incl. claims on Trust Fund (TCMFs) & 31,653 & 34,616 & 35,134 & 42,333 & 45,332 \\
\hline TCMFs & 11,188 & 11,188 & 11,188 & 11,188 & 11,188 \\
\hline Stock of domestic public debt & 20,465 & 23,428 & 23,946 & 31,145 & 34,144 \\
\hline Banking sector & 13,268 & 16,422 & 15,903 & 17,488 & 20,044 \\
\hline Bank of Cape Verde & 4,547 & 5,590 & 4,776 & 4,779 & 4,779 \\
\hline Banco Comercial do Atlântico & 5,131 & 6,521 & 7,253 & 9,298 & 10,643 \\
\hline Caixa Económica de Cabo Verde & 2,200 & 1,981 & 1,331 & 1,030 & 2,104 \\
\hline Banco Totta e Açores & 460 & 963 & 887 & 963 & 620 \\
\hline Caixa Geral de Depositos & 930 & 1,368 & 1,656 & 1,419 & 1,898 \\
\hline Nonbanking sector & 3,936 & 5,933 & 6,342 & 7,304 & 7,876 \\
\hline INPS ${ }^{1}$ & 2,584 & 5,295 & 5,571 & 6,537 & 7,374 \\
\hline Garantia & 125 & 128 & 145 & 150 & 53 \\
\hline Other & 1,227 & 511 & 626 & 617 & 449 \\
\hline
\end{tabular}

Sources: Ministry of Finance and Public Administration, Bank of Cape Verde, and staff estimates.

${ }^{1}$ Instituto Nacional de Previdencia Social (INPS). 
Table 12. Cape Verde: Monetary Survey, 2001-05

\begin{tabular}{|c|c|c|c|c|c|c|}
\hline & 2000 & 2001 & 2002 & 2003 & 2004 & 2005 \\
\hline & \multicolumn{6}{|c|}{ (Millions of Cape Verde escudos) } \\
\hline Net foreign assets & 7,139 & 9,557 & 11,331 & 10,456 & 13,787 & 21,889 \\
\hline Foreign assets & 9,244 & 11,489 & 13,841 & 13,434 & 16,912 & 27,549 \\
\hline Of which: foreign reserves & 3,349 & 5,238 & 8,391 & 8,173 & 11,296 & 16,260 \\
\hline Foreign liabilities & $-2,104$ & $-1,932$ & $-2,511$ & $-2,978$ & $-3,125$ & $-5,660$ \\
\hline Net domestic assets & 34,561 & 36,239 & 41,027 & 46,428 & 49,082 & 50,755 \\
\hline Net domestic credit & 41,184 & 44,093 & 49,916 & 54,503 & 57,319 & 59,404 \\
\hline Net claims on general government & 21,702 & 21,665 & 24,790 & 25,561 & 25,684 & 24,863 \\
\hline Claims on the Trust Fund (TCMFs) & 10,600 & 10,600 & 10,600 & 11,038 & 11,038 & 11,038 \\
\hline Net claims on the central government & 11,625 & 11,676 & 14,368 & 14,858 & 15,066 & 14,418 \\
\hline Credit to central government & 13,082 & 13,356 & 16,546 & 17,097 & 17,485 & 20,044 \\
\hline Deposits of central government & $-1,457$ & $-1,680$ & $-2,178$ & $-2,239$ & $-2,419$ & $-5,626$ \\
\hline Of which: project deposits & -211 & -331 & -397 & -393 & -277 & -351 \\
\hline Net claims on local government & -57 & 3 & 8 & 67 & 75 & -24 \\
\hline Net claims on other government agencies (INPS) & -466 & -614 & -186 & -402 & -495 & -569 \\
\hline Credit to the economy & 19,483 & 22,428 & 25,126 & 28,943 & 31,635 & 34,541 \\
\hline Credit to public enterprises & 156 & 215 & 230 & 180 & 161 & 476 \\
\hline Credit to private sector & 19,327 & 22,206 & 24,890 & 28,726 & 31,430 & 34,040 \\
\hline Claims on nonbank financial institutions & 0 & 7 & 6 & 37 & 44 & 26 \\
\hline Other items (net) & $-6,624$ & $-7,854$ & $-8,889$ & $-8,075$ & $-8,237$ & $-8,648$ \\
\hline Broad money (M2) & 41,700 & 45,796 & 52,358 & 56,884 & 62,870 & 72,644 \\
\hline Narrow money (M1) & 20,131 & 20,759 & 22,621 & 22,989 & 24,426 & 28,719 \\
\hline Currency outside banks & 6,458 & 6,703 & 6,459 & 6,516 & 6,765 & 7,634 \\
\hline Demand deposits & 13,673 & 14,056 & 16,162 & 16,474 & 17,661 & 21,086 \\
\hline Quasi money & 19,371 & 22,931 & 27,636 & 31,662 & 35,762 & 40,566 \\
\hline Time deposits & 18,081 & 21,802 & 25,857 & 29,983 & 34,313 & 38,390 \\
\hline Other quasi-monetary deposits & 1,290 & 1,130 & 1,779 & 1,679 & 1,449 & 2,176 \\
\hline \multirow[t]{2}{*}{ Foreign currency deposits } & 2,198 & 2,106 & 2,101 & 2,232 & 2,682 & 3,359 \\
\hline & \multicolumn{6}{|c|}{ (Percent of recurrent expenditure) } \\
\hline Net foreign assets & -2.6 & 5.8 & 3.9 & -1.7 & 5.9 & 12.9 \\
\hline Net domestic assets & 15.5 & 4.0 & 10.5 & 10.3 & 4.7 & 2.7 \\
\hline Net domestic credit & 22.5 & 7.0 & 12.7 & 8.8 & 5.0 & 3.3 \\
\hline Net claims on the central government & 11.1 & 0.1 & 5.9 & 0.9 & 0.4 & -1.0 \\
\hline Credit to the economy & 1.6 & 7.1 & 5.9 & 7.3 & 4.7 & 4.6 \\
\hline Credit to public enterprises & -1.0 & 0.1 & 0.0 & -0.1 & 0.0 & 0.5 \\
\hline Credit to private sector & 2.5 & 6.9 & 5.9 & 7.3 & 4.8 & 4.2 \\
\hline Other items (net) & -7.0 & -2.9 & -2.3 & 1.6 & -0.3 & -0.7 \\
\hline \multicolumn{7}{|l|}{ Selected monetary indicators } \\
\hline Income velocity of money & 1.64 & 1.59 & 1.48 & 1.46 & 1.37 & 1.29 \\
\hline Emigrant deposits & 12,477 & 15,585 & 19,042 & 22,153 & 25,091 & 28,318 \\
\hline Excess reserves /total deposits (percent) & 0.4 & 0.5 & 1.1 & 0.8 & 1.0 & 2.1 \\
\hline Money multilplier (M2/M0) & 3.08 & 3.14 & 3.22 & 3.29 & 3.40 & 3.44 \\
\hline Credit to the economy (percentage change) & 3.0 & 15.1 & 12.0 & 15.2 & 9.3 & 9.2 \\
\hline
\end{tabular}

Sources: Bank of Cape Verde, and staff estimates and projections. 
Table 13. Cape Verde: Summary Accounts of the Bank of Cape Verde, 2001-05 (Millions of Cape Verde escudos, unless otherwise indicated)

\section{Bank of Cape Verde}

Net foreign assets

Of which: net international reserves

Foreign assets

Foreign liabilities

Short-term foreign liabilities

Medium- and long-term foreign liabilities

Net domestic assets

Trust Fund claims

Net claims on central government

Credit to central government

Deposits of central government

Of which: project accounts

Of which: foreign currency deposits

Claims on local government

Credit to the economy

Credit to public enterprises

Claims on nonbank financial institutions

Credit to commercial banks

Other items (net)

Assets

Liabilities

Reserve money (M0)

Currency outside banks

Cash in vaults

Deposits of commercial banks

Deposits of private sector

Deposits of other financial institutions

Deposits of public sector (local govt and PSE)

Gross international reserves (millions of euros)

Net international reserves (million of euros)

Reserve money (12-month change in percent)
Net domestic credit

Credit to private sector

\begin{tabular}{|c|c|c|c|c|c|}
\hline 4,053 & 5,945 & 8,632 & 8,088 & 10,698 & 15,308 \\
\hline 3,221 & 5,100 & 7,984 & 7,528 & 10,524 & 15,109 \\
\hline 4,212 & 6,115 & 9,135 & 8,818 & 11,526 & 16,523 \\
\hline-160 & -170 & -503 & -730 & -828 & $-1,215$ \\
\hline-128 & -138 & -407 & -645 & -772 & $-1,151$ \\
\hline-32 & -32 & -96 & -85 & -57 & -63 \\
\hline 9,499 & 8,635 & 7,605 & 9,201 & 7,790 & 5,828 \\
\hline 10,867 & 9,976 & 9,728 & 10,651 & 9,439 & 6,889 \\
\hline 4,167 & 4,167 & 4,167 & 4,605 & 4,605 & 4,605 \\
\hline 4,444 & 4,183 & 4,103 & 4,424 & 3,594 & 1,093 \\
\hline 5,043 & 4,857 & 5,302 & 5,373 & 4,779 & 4,779 \\
\hline-598 & -675 & $-1,199$ & -948 & $-1,184$ & $-3,685$ \\
\hline-211 & -331 & -397 & -393 & -277 & -351 \\
\hline-272 & -256 & -330 & -456 & -426 & $-1,347$ \\
\hline & 0 & 0 & 0 & 0 & 0 \\
\hline 1,157 & 1,189 & 1,184 & 1,212 & 1,196 & 1,163 \\
\hline 82 & 82 & 72 & 72 & 61 & 54 \\
\hline 1,075 & 1,106 & 1,112 & 1,107 & 1,106 & 1,094 \\
\hline 0 & 0 & 0 & 33 & 30 & 15 \\
\hline 1,099 & 438 & 275 & 410 & 44 & 27 \\
\hline$-1,368$ & $-1,341$ & $-2,123$ & $-1,451$ & $-1,649$ & $-1,061$ \\
\hline 2,276 & 2,616 & 2,345 & 2,396 & 2,438 & 2,482 \\
\hline 3,644 & 3,957 & 4,468 & 3,847 & 4,086 & 3,543 \\
\hline 13,552 & 14,581 & 16,237 & 17,289 & 18,489 & 21,136 \\
\hline 6,458 & 6,703 & 6,459 & 6,516 & 6,765 & 7,634 \\
\hline 597 & 650 & 1,013 & 808 & 1,072 & 1,058 \\
\hline 6,496 & 7,227 & 8,764 & 9,964 & 10,630 & 12,443 \\
\hline 0 & 0 & 0 & 0 & 0 & 0 \\
\hline 1 & 1 & 1 & 0 & 21 & 2 \\
\hline 30.4 & 47.5 & 76.1 & 74.1 & 102.4 & 147.5 \\
\hline 29.2 & 46.3 & 72.4 & 68.3 & 95.4 & 137.0 \\
\hline 15.8 & 7.6 & 11.4 & 6.5 & 6.9 & 14.3 \\
\hline
\end{tabular}

Sources: Bank of Cape Verde, and staff estimates and projections. 
Table 14. Cape Verde: Summary Accounts of Commercial Banks, 2001-05

(Millions of Cape Verde escudos, unless otherwise indicated)

\begin{tabular}{|c|c|c|c|c|c|c|}
\hline \multicolumn{7}{|l|}{ Commercial banks } \\
\hline Net foreign assets & 3,086 & 3,612 & 2,699 & 2,368 & 3,089 & 6,581 \\
\hline Foreign assets & 5,031 & 5,374 & 4,706 & 4,616 & 5,386 & 11,026 \\
\hline Foreign liabilities & $-1,945$ & $-1,762$ & $-2,007$ & $-2,248$ & $-2,297$ & $-4,445$ \\
\hline of which: Nonresident deposits & $-1,551$ & $-1,353$ & $-1,650$ & $-1,847$ & $-2,022$ & $-3,520$ \\
\hline Net domestic assets & 32,155 & 35,481 & 43,199 & 48,000 & 52,994 & 58,428 \\
\hline Net domestic credit & 37,410 & 41,993 & 49,964 & 54,625 & 59,582 & 66,016 \\
\hline Net claims on general government & 13,091 & 13,316 & 16,521 & 16,531 & 17,485 & 19,165 \\
\hline Trust Fund claims & 6,433 & 6,433 & 6,433 & 6,433 & 6,433 & 6,433 \\
\hline Other government deposits (INPS) & -466 & -614 & -186 & -402 & -495 & -569 \\
\hline Net claims on central government & 7,180 & 7,493 & 10,265 & 10,433 & 11,472 & 13,324 \\
\hline Loans and overdrafts & 5,056 & 5,483 & 6,704 & 7,302 & 7,872 & 9,657 \\
\hline Holding of government securities & 2,983 & 3,015 & 4,540 & 4,423 & 4,835 & 5,609 \\
\hline Deposits of central government & -859 & $-1,005$ & -979 & $-1,291$ & $-1,234$ & $-1,941$ \\
\hline Net claims on local government & -57 & 3 & 8 & 67 & 75 & -24 \\
\hline Claims on local government & 289 & 261 & 266 & 239 & 209 & 232 \\
\hline Deposits of local government & -347 & -258 & -259 & -172 & -134 & -256 \\
\hline Credit to the economy & 18,326 & 21,239 & 23,941 & 27,731 & 30,439 & 33,378 \\
\hline Credit to public enterprises & 73 & 133 & 158 & 108 & 101 & 422 \\
\hline Credit to private sector & 18,252 & 21,099 & 23,778 & 27,619 & 30,325 & 32,945 \\
\hline Claims on nonbank financial institutions & 0 & 7 & 6 & 4 & 14 & 11 \\
\hline Net claims on the Bank of Cape Verde & 5,994 & 7,438 & 9,502 & 10,363 & 11,658 & 13,473 \\
\hline Total reserves & 7,093 & 7,877 & 9,777 & 10,772 & 11,702 & 13,500 \\
\hline Vault cash & 597 & 650 & 1,013 & 808 & 1,072 & 1,058 \\
\hline Deposits with central bank & 6,496 & 7,227 & 8,764 & 9,964 & 10,630 & 12,443 \\
\hline Required reserves & 6,343 & 7,037 & 8,262 & 9,570 & 10,095 & 11,052 \\
\hline Excess reserves & 153 & 190 & 503 & 394 & 535 & 1,391 \\
\hline Credit to the Bank of Cape Verde & $-1,099$ & -438 & -275 & -410 & -44 & -27 \\
\hline Other items (net) & $-5,256$ & $-6,513$ & $-6,765$ & $-6,625$ & $-6,588$ & $-7,587$ \\
\hline Deposit liabilities to nonbank residents & 35,241 & 39,092 & 45,898 & 50,368 & 56,083 & 65,009 \\
\hline Local currency deposits & 33,043 & 36,987 & 43,796 & 48,135 & 53,402 & 61,650 \\
\hline Demand deposits & 13,672 & 14,056 & 16,161 & 16,473 & 17,640 & 21,084 \\
\hline Of which: emigrant deposits & 1,387 & 1,765 & 2,133 & 2,202 & 2,591 & 3,394 \\
\hline Quasi money & 19,371 & 22,931 & 27,636 & 31,662 & 35,762 & 40,566 \\
\hline Time deposits & 18,081 & 21,802 & 25,857 & 29,983 & 34,313 & 38,390 \\
\hline Of which: emigrant deposits & 10,015 & 12,672 & 15,817 & 18,918 & 21,521 & 23,825 \\
\hline Other quasi-monetary deposits & 1,290 & 1,130 & 1,779 & 1,679 & 1,449 & 2,176 \\
\hline Foreign currency deposits & 2,198 & 2,106 & 2,101 & 2,232 & 2,682 & 3,359 \\
\hline Of which: emigrant deposits & 1,075 & 1,148 & 1,092 & 1,033 & 979 & 1,100 \\
\hline \multicolumn{7}{|l|}{ Memorandum items: } \\
\hline Emigrant deposits (ratio to total deposits) & 0.30 & 0.34 & 0.36 & 0.39 & 0.40 & 0.39 \\
\hline Other deposits (ratio to total deposits) & 0.70 & 0.66 & 0.64 & 0.61 & 0.60 & 0.61 \\
\hline Composition of emigrant deposits & 1.00 & 1.00 & 1.00 & 1.00 & 1.00 & 1.00 \\
\hline Local currency & 0.91 & 0.93 & 0.94 & 0.95 & 0.96 & 0.96 \\
\hline Demand & 0.11 & 0.11 & 0.11 & 0.10 & 0.10 & 0.12 \\
\hline Time & 0.80 & 0.81 & 0.83 & 0.85 & 0.86 & 0.84 \\
\hline Foreign currency & 0.09 & 0.07 & 0.06 & 0.05 & 0.04 & 0.04 \\
\hline
\end{tabular}

Sources: Bank of Cape Verde, and staff estimates and projections. 
Table 15. Cape Verde: Interest Rate Structure, 2001-05

(Annual average, percent)

\begin{tabular}{lrrrrr}
\hline & 2001 & 2002 & 2003 & 2004 & 2005 \\
\hline & & & & & \\
Refinance rate & 8.5 & 8.5 & 8.5 & 8.5 & 8.5 \\
Average T-bill rates & 9.7 & 8.3 & 5.9 & 6.5 & 4.1 \\
$\quad 91$ days & 9.5 & 7.9 & 5.8 & 6.2 & 4.2 \\
182 days & 10.3 & 8.0 & 5.8 & 6.4 & 4.1 \\
364 days & 9.2 & 9.0 & 6.2 & 6.9 & 4.1 \\
& & & & & \\
Lending rates (average) & 13.8 & 14.1 & 13.4 & 13.4 & 13.0 \\
Up to 90 days & 12.8 & 13.2 & 12.7 & 12.7 & 12.3 \\
From 91 to 180 days & 13.3 & 13.5 & 13.1 & 13.1 & 12.6 \\
From 181 days to one year & 13.6 & 13.9 & 13.3 & 13.4 & 12.9 \\
From one to two years & 14.4 & 15.1 & 14.3 & 14.2 & 13.7 \\
From two to five years & 14.2 & 14.6 & 13.6 & 13.6 & 13.1 \\
$\quad$ More than five years & 14.3 & 14.5 & 13.6 & 13.6 & 13.2 \\
& & & & & \\
Time deposit rates & & & & & \\
$\quad$ Up to 60 days & 6.1 & 6.2 & 5.1 & 4.7 & 4.4 \\
From 61 to 90 days & 4.3 & 4.6 & 4.1 & 4.0 & 3.6 \\
From 91 to 180 days & 4.7 & 4.9 & 3.9 & 3.5 & 3.3 \\
$\quad$ From 181 days to one year & 6.1 & 6.2 & 5.2 & 4.4 & 4.3 \\
$\quad$ More than one year & 7.5 & 7.5 & 6.2 & 5.7 & 5.3 \\
Emigrants time deposit rates & & & & & \\
From 30 to 180 days & & & & & \\
From 181 days to one year & 6.8 & 7.9 & 6.2 & 5.5 & 5.0 \\
$\quad$ More than one year & 9.0 & 8.7 & 7.2 & 6.6 & 5.7 \\
& 9.8 & 9.6 & 8.1 & 7.9 & 6.5 \\
\hline
\end{tabular}

Source: Bank of Cape Verde. 
Table 16. Cape Verde: Balance of Payments, 2001-05

(Millions of Cape Verde escudos, unless otherwise indicated)

\begin{tabular}{|c|c|c|c|c|c|}
\hline & 2001 & 2002 & 2003 & 2004 & 2005 \\
\hline Current account balance (including official transfers) & $-7,352$ & $-8,328$ & $-8,790$ & $-11,799$ & $-3,980$ \\
\hline Trade balance & $-23,958$ & $-27,693$ & $-30,065$ & $-33,656$ & $-30,960$ \\
\hline Exports, f.o.b. & 4,577 & 4,909 & 5,150 & 5,093 & 7,891 \\
\hline Imports, f.o.b. & $-28,535$ & $-32,602$ & $-35,215$ & $-38,749$ & $-38,851$ \\
\hline Services (net) & 1,254 & 1,285 & 1,347 & 2,743 & 5,149 \\
\hline Credit & 15,981 & 18,554 & 19,736 & 21,096 & 23,668 \\
\hline Of which: tourism & 6,539 & 6,881 & 8,306 & 8,496 & 9,566 \\
\hline Debit & $-14,727$ & $-17,270$ & $-18,389$ & $-18,353$ & $-18,519$ \\
\hline Income (net) & -577 & $-1,701$ & $-1,258$ & $-1,570$ & $-2,966$ \\
\hline Of which: Government interest payments & -478 & -719 & -514 & -549 & -551 \\
\hline Current transfers (net) & 15,929 & 19,782 & 21,187 & 20,685 & 24,797 \\
\hline Official & 2,501 & 4,159 & 4,776 & 4,697 & 4,090 \\
\hline Private & 13,428 & 15,623 & 16,411 & 15,989 & 20,706 \\
\hline Capital and financial account (net) & 8,970 & 12,233 & 7,204 & 9,584 & 7,173 \\
\hline Capital transfers & 2,644 & 1,824 & 2,481 & 2,089 & 1,821 \\
\hline Direct investment (net) & 2,079 & 1,126 & 1,531 & 1,812 & 1,708 \\
\hline Net official flows & -499 & 647 & 1,448 & 517 & 2,080 \\
\hline Of which: Amortization & $-2,435$ & $-2,182$ & $-2,101$ & $-2,403$ & $-2,234$ \\
\hline Other capital & 3,410 & 8,085 & 2,281 & 5,166 & 1,763 \\
\hline Commercial banks & -2 & 15 & -602 & -536 & $-2,696$ \\
\hline Emigrant deposit flows & 3,108 & 3,457 & 3,111 & 2,938 & 3,227 \\
\hline Commercial credit (net) & 663 & 2,577 & -603 & 203 & 564 \\
\hline Other & -358 & 2,036 & 375 & 2,561 & 669 \\
\hline Net errors and omissions & 621 & 685 & 1,052 & 5,541 & 1,808 \\
\hline Overall balance & 2,239 & 4,590 & -534 & 3,327 & 5,000 \\
\hline Financing & $-2,239$ & $-4,590$ & 534 & $-3,327$ & $-5,000$ \\
\hline Gross international reserves ( $-=$ accumulation) & $-2,331$ & $-2,792$ & 102 & $-3,338$ & $-4,964$ \\
\hline Of which: IMF (net) & 0 & 354 & 291 & 127 & 380 \\
\hline Exceptional financing & 93 & $-1,798$ & 432 & 11 & -36 \\
\hline \multicolumn{6}{|l|}{ Memorandum items: } \\
\hline Current account (including official transfers) ${ }^{1}$ & -10.6 & -11.4 & -11.1 & -14.4 & -4.6 \\
\hline Current account (excluding official transfers) ${ }^{1}$ & -14.2 & -17.2 & -17.1 & -20.1 & -9.3 \\
\hline Overall balance $^{1}$ & 3.2 & 6.3 & -0.7 & 4.1 & 5.7 \\
\hline Gross international reserves & 5,238 & 8,391 & 8,173 & 11,296 & 16,260 \\
\hline In months of next year's import of goods and service & 1.3 & 1.9 & 1.7 & 2.4 & 3.0 \\
\hline External public debt & 39,202 & 41,068 & 45,724 & 44,362 & 48,303 \\
\hline Exchange rate per U.S. dollar (average) & 123.2 & 117.2 & 97.7 & 88.8 & 88.7 \\
\hline
\end{tabular}

Sources: Bank of Cape Verde, and staff estimates and projections.

${ }^{1}$ Percent of GDP. 
Table 17. Cape Verde: Merchandise Exports, 2001-2005

(Millions of Cape Verde escudos)

\begin{tabular}{lrrrrr}
\hline & 2001 & 2002 & 2003 & 2004 & 2005 \\
\hline Agricultural products & 10.9 & 0.0 & 3.4 & 0.0 & 0.0 \\
& & & & & \\
& 37.7 & 58.8 & 48.2 & 109.0 & 635.0 \\
Fish and crustaceans & 27.0 & 20.1 & 8.9 & 41.6 & 600.6 \\
$\quad$ Fish (including tuna) & 9.2 & 4.0 & 6.6 & 5.4 & 4.9 \\
$\quad$ Canned & 17.3 & 12.9 & 0.9 & 35.2 & 159.3 \\
$\quad$ Frozen & 0.5 & 3.2 & 1.4 & 1.0 & 436.4 \\
$\quad$ Fresh & 10.7 & 38.7 & 39.3 & 67.4 & 34.4 \\
Shellfish and lobster & & & & & \\
& 2.9 & 57.0 & 1.6 & 0.0 & 0.0 \\
Hides and skin & 511.2 & 445.4 & 381.8 & 415.9 & 245.0 \\
& & & & & \\
Shoes and shoe parts & 1456.8 & 645.7 & 801.1 & 809.4 & 691.4 \\
& 606.5 & 574.5 & 674.1 & 766.3 & 614.3 \\
Miscellaneous & 26.4 & 11.3 & 0.0 & 0.0 & 0.0 \\
$\quad$ Clothing & 12.3 & 59.9 & 127.0 & 43.1 & 77.1 \\
$\quad$ Electronic components & 1207.8 & 1234.7 & 1236.0 & 1334.3 & 1571.4 \\
$\quad$ Other & & & & & \\
Total national exports & 2955.1 & 3315.4 & 3574.1 & 3517.4 & 6039.2 \\
Total reexports & 4162.9 & 4550.1 & 4810.1 & 4851.7 & 7610.6 \\
Total exports & & & & & \\
\hline
\end{tabular}

Sources: Bank of Cape Verde, Directorate of Customs, National Statistics Institute, and staff estimates. 
Table 18. Cape Verde: Merchandise Imports, c.i.f., 2001-2005

(Millions of Cape Verde escudos, unless otherwise indicated)

\begin{tabular}{|c|c|c|c|c|c|}
\hline & 2001 & 2002 & 2003 & 2004 & 2005 \\
\hline Consumer goods & 12,571 & 12,732 & 13,403 & 14,503 & 16,042 \\
\hline Primary food products & 1,356 & 1,809 & 1,847 & 1,497 & 1,717 \\
\hline Intermediate food products & 7,132 & 6,565 & 7,006 & 8,370 & 8,819 \\
\hline Transport materials & 1,418 & 1,167 & 1,269 & 1,068 & 1,177 \\
\hline Other durable goods & 753 & 1,008 & 1,024 & 1,355 & 1,871 \\
\hline Other semidurable goods & 775 & 919 & 906 & 867 & 843 \\
\hline Other nondurable goods & 1,137 & 1,265 & 1,353 & 1,345 & 1,615 \\
\hline Intermediary goods & 6,027 & 7,535 & 7,137 & 8,554 & 9,553 \\
\hline Primary food products & 195 & 208 & 198 & 710 & 284 \\
\hline Intermediate food products & 89 & 134 & 127 & 124 & 214 \\
\hline Other primary products & 111 & 81 & 92 & 95 & 117 \\
\hline Agricultural products & 16 & 11 & 18 & 21 & 21 \\
\hline Finished food products & 124 & 134 & 135 & 144 & 118 \\
\hline Shoe products & 103 & 142 & 97 & 125 & 135 \\
\hline Other industrial products & 235 & 293 & 301 & 277 & 345 \\
\hline Construction materials & 2,326 & 3,025 & 3,069 & 3,915 & 4,578 \\
\hline Of which: cement & 976 & 1,164 & 1,315 & 1,467 & 1,737 \\
\hline metal & 714 & 1,034 & 1,170 & 1,424 & 1,473 \\
\hline Wood products & 783 & 676 & 964 & 893 & 1,017 \\
\hline Electrical materials & 659 & 1,401 & 614 & 642 & 769 \\
\hline Other intermediate products & 1,205 & 1,253 & 1,418 & 1,426 & 1,748 \\
\hline Parts and accessories for transport materials & 181 & 178 & 104 & 182 & 207 \\
\hline Capital goods & 4,560 & 6,220 & 4,647 & 5,172 & 6,536 \\
\hline Machines & 2,589 & 2,944 & 2,218 & 2,883 & 3,296 \\
\hline Of which: Agriculture & 13 & 27 & 10 & 40 & 20 \\
\hline Industry & 105 & 182 & 263 & 126 & 318 \\
\hline Construction & 160 & 98 & 122 & 209 & 222 \\
\hline Telecommunication & 162 & 118 & 124 & 298 & 203 \\
\hline Transportation & 1,349 & 2,112 & 2,006 & 1,942 & 2,770 \\
\hline Cars & 0 & 494 & 321 & 321 & 277 \\
\hline Air planes & 2 & 0 & 1 & 0 & 0 \\
\hline Ships & 22 & 415 & 355 & 540 & 1,170 \\
\hline Tractors & 423 & 933 & 1,068 & 778 & 915 \\
\hline Engines & 623 & 1,164 & 424 & 347 & 470 \\
\hline Petroleum imports & 1,520 & 1,635 & 4,329 & 2,862 & 2,691 \\
\hline Of which: fuel oil & 274 & 192 & 515 & 607 & 549 \\
\hline diesel oil & 882 & 950 & 2,355 & 1,359 & 1,891 \\
\hline gasoline & 154 & 319 & 268 & 262 & 251 \\
\hline kerosene & 0 & 11 & 0 & 0 & 0 \\
\hline Other imports & 5,297 & 4,147 & 4,697 & 3,237 & 3,422 \\
\hline Total merchandise imports, c.i.f. & 29,974 & 32,269 & 34,213 & 34,326 & 38,245 \\
\hline Total merchandise imports, f.o.b. & 28,535 & 32,583 & 33,403 & 31,293 & 34,749 \\
\hline Ratio of imports c.i.f. to imports f.o.b. ${ }^{1}$ & 105 & 99 & 102 & 110 & 110 \\
\hline
\end{tabular}

Source: Cape Verde authorities.

${ }^{1}$ In percent. 
Table 19. Cape Verde: International Trade in Services, 2001-05

(Millions of Cape Verde escudos)

\begin{tabular}{|c|c|c|c|c|c|}
\hline & 2001 & 2002 & 2003 & 2004 & 2005 \\
\hline Total services (net) & $1,253.6$ & $1,284.6$ & $1,346.6$ & $2,742.8$ & $5,247.9$ \\
\hline Credit & $15,980.7$ & $18,554.2$ & $19,735.8$ & $21,095.7$ & 23767.2 \\
\hline Transport & $6,244.0$ & $8,674.3$ & $8,108.6$ & $8,792.5$ & 9399.7 \\
\hline Sea & 399.0 & 205.8 & 191.6 & 330.2 & 600.5 \\
\hline Air & $5,844.9$ & $8,468.5$ & $7,917.0$ & $8,462.3$ & 8799.1 \\
\hline Travel & $6,621.5$ & $7,007.4$ & $8,453.9$ & $8,725.3$ & 9963.2 \\
\hline Business & 82.4 & 126.7 & 147.8 & 229.4 & 397.2 \\
\hline Personal & $6,539.1$ & $6,880.7$ & $8,306.1$ & $8,495.9$ & 9566.0 \\
\hline Tourism & $6,539.1$ & $6,880.7$ & $8,306.1$ & $8,495.6$ & 9565.9 \\
\hline Communications & $1,334.3$ & $1,474.4$ & $1,425.3$ & $1,516.4$ & 1677.8 \\
\hline Construction & 19.9 & 7.4 & 8.0 & 0.0 & 0.0 \\
\hline Insurance & 304.1 & 36.2 & 115.4 & 153.5 & 145.3 \\
\hline Financial services & 4.4 & 8.5 & 14.2 & 5.5 & 58.3 \\
\hline Information services & 11.5 & 3.3 & 4.2 & 9.1 & 1.5 \\
\hline Royalties and licences & 16.1 & 68.5 & 7.2 & 0.0 & 0.0 \\
\hline Other & $1,424.9$ & $1,274.3$ & $1,598.9$ & $1,893.4$ & 2521.4 \\
\hline Debit & $-14,727.1$ & $-17,269.5$ & $-18,389.2$ & $-18,352.9$ & -18519.3 \\
\hline Transport & $-6,183.8$ & $-8,001.1$ & $-8,787.4$ & $-8,083.1$ & -8686.5 \\
\hline Sea & $-3,090.7$ & $-3,216.3$ & $-3,092.6$ & $-2,939.4$ & -3484.5 \\
\hline Air & $-3,093.1$ & $-4,784.8$ & $-5,694.8$ & $-5,143.7$ & -5202.0 \\
\hline Travel & $-5,877.4$ & $-6,690.3$ & $-7,079.8$ & $-6,899.8$ & -5953.6 \\
\hline Business & -647.5 & -866.1 & -913.3 & -645.5 & -1030.0 \\
\hline Personal & $-5,229.9$ & $-5,824.2$ & $-6,166.5$ & $-6,254.3$ & -4923.6 \\
\hline Tourism & $-4,005.6$ & $-4,312.1$ & $-4,438.8$ & $-3,913.4$ & -3489.8 \\
\hline Communications & -524.7 & -560.1 & -338.5 & -257.5 & -298.0 \\
\hline Construction & 0.0 & -0.2 & 0.0 & 0.0 & -0.3 \\
\hline Insurance & -455.0 & -524.0 & -510.1 & -641.5 & -672.1 \\
\hline Financial services & -342.4 & -86.3 & -185.6 & -226.0 & -346.4 \\
\hline Information services & -240.1 & -289.1 & -289.2 & -336.4 & -420.1 \\
\hline Royalties and licences & -16.6 & -23.2 & -4.5 & -23.1 & -6.1 \\
\hline Other & -1086.9 & -1095.4 & -1194.0 & -1885.5 & -2136.2 \\
\hline
\end{tabular}

Source: Bank of Cape Verde. 
Table 20. Cape Verde: Direction of Trade, 2001-05

(Percent of total)

\begin{tabular}{|c|c|c|c|c|c|}
\hline & 2001 & 2002 & 2003 & 2004 & 2005 \\
\hline Exports & 100.0 & 100.0 & 100.0 & 100.0 & 100.0 \\
\hline France & 0.1 & 21.9 & 25.9 & 1.1 & 1.2 \\
\hline Germany & 0.6 & 0.8 & 1.9 & 1.7 & 2.8 \\
\hline Netherlands & 0.2 & 0.1 & 0.5 & 0.2 & 1.5 \\
\hline Italy & 0.0 & 0.3 & 0.2 & 0.1 & 1.4 \\
\hline Portugal & 79.1 & 36.2 & 31.5 & 59.0 & 34.5 \\
\hline Spain & 0.2 & 0.1 & 0.2 & 0.7 & 39.6 \\
\hline United Kingdom & 0.1 & 24.9 & 17.0 & 11.3 & 3.7 \\
\hline United States & 17.7 & 7.8 & 18.5 & 17.1 & 9.5 \\
\hline Other & 2.0 & 7.9 & 4.3 & 8.7 & 5.7 \\
\hline Imports & 100.0 & 100.0 & 100.0 & 100.0 & 100.0 \\
\hline Belgium & 3.8 & 3.0 & 3.9 & 3.4 & 4.6 \\
\hline Brazil & 2.3 & 1.8 & 2.7 & 4.1 & 4.3 \\
\hline China, P.R. & 0.9 & 0.6 & 0.8 & 0.7 & 1.2 \\
\hline Côte d'Ivoire & 0.8 & 2.8 & 1.3 & 0.4 & 2.3 \\
\hline France & 3.3 & 3.4 & 4.2 & 2.5 & 4.8 \\
\hline Germany & 3.2 & 5.5 & 2.2 & 1.8 & 1.6 \\
\hline Italy & 3.1 & 3.5 & 3.2 & 4.2 & 8.0 \\
\hline Netherlands & 7.3 & 6.8 & 9.1 & 8.4 & 7.0 \\
\hline Portugal & 45.8 & 46.3 & 47.2 & 42.1 & 41.4 \\
\hline Senegal & 0.4 & 1.9 & 0.5 & 0.4 & 0.4 \\
\hline Spain & 4.2 & 3.7 & 3.6 & 5.3 & 5.5 \\
\hline United Kingdom & 1.7 & 2.9 & 2.9 & 1.4 & 1.8 \\
\hline United States & 3.2 & 3.2 & 2.8 & 12.4 & 2.2 \\
\hline Other & 20.1 & 14.8 & 15.6 & 12.9 & 15.0 \\
\hline
\end{tabular}

Source: Direction of Trade Statistics, IMF. 


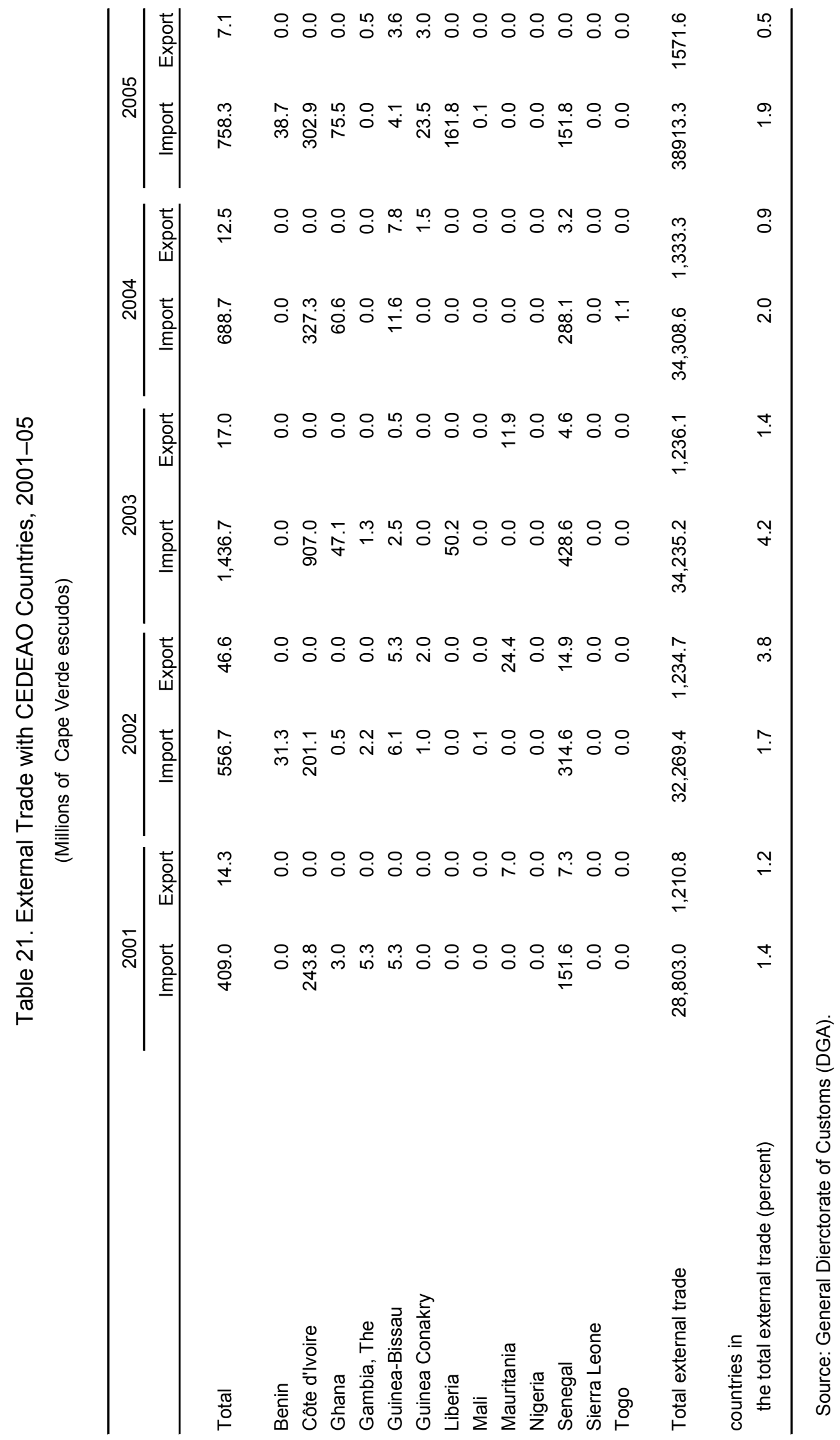


Table 22. Cape Verde: Primary Income from Abroad, 2001-05

(Millions of Cape Verde escudos)

\begin{tabular}{lrrrrr}
\hline & 2001 & 2002 & 2003 & 2004 & 2005 \\
\hline & & & & & \\
Total primary income (net) & -576.7 & $-1,701.3$ & $-1,258.4$ & $-1,570.4$ & -2965.7 \\
& & & & & \\
Credit & 831.0 & 834.3 & $1,599.2$ & $1,601.7$ & 1649.2 \\
$\quad$ Investment income & 684.8 & 788.5 & $1,582.7$ & $1,567.3$ & 1636.6 \\
$\quad$ Of which: Trust Fund & 402.5 & 503.4 & 508.4 & 638.6 & 363.3 \\
Compensation of employees & 146.2 & 45.8 & 16.5 & 34.4 & 12.5 \\
& & & & $-3,172.1$ & -4614.9 \\
Debit & $-1,407.7$ & $-2,535.5$ & $-2,857.5$ & $-4,117.6$ & -4502.7 \\
Investment income & $-1,373.1$ & $-2,504.5$ & $-2,803.9$ & -549.4 & -550.8 \\
$\quad$ Goverment interest & -478.1 & -719.3 & -514.4 & $-2,5$ & $-3,951.9$ \\
$\quad$ Other investment income & -895.0 & $-1,785.2$ & $-2,289.5$ & $-2,568.2$ & -112.2 \\
$\quad$ Compensation of employees & -34.6 & -31.0 & -53.7 & -54.5 & \\
\hline
\end{tabular}

Source: Bank of Cape Verde. 
Table 23. Cape Verde: Private and Government Transfers, 2001-05 (Millions of Cape Verde escudos)

\begin{tabular}{lrrrrr}
\hline & 2001 & 2002 & 2003 & 2004 & 2005 \\
\hline Current transfers (net) & & & & & \\
& $15,928.8$ & $19,781.9$ & $21,187.1$ & $20,685.4$ & 24796.7 \\
Private (net) & & & & & \\
$\quad$ Credit & $13,428.1$ & $15,623.0$ & $16,410.9$ & $15,988.6$ & 20706.4 \\
$\quad$ Emigrant remittances & $16,478.5$ & $18,022.7$ & $18,096.9$ & $19,713.8$ & 23286.4 \\
$\quad$ Other transfers & $8,851.8$ & $9,126.8$ & $9,514.0$ & $8,750.8$ & 11018.4 \\
Debit & $7,626.6$ & $8,896.0$ & $8,582.8$ & $10,963.1$ & 12268.1 \\
Remittances & $-3,050.4$ & $-2,399.7$ & $-1,685.9$ & $-3,725.2$ & -2580.1 \\
$\quad$ Other & -352.9 & -571.7 & -646.5 & $-1,005.4$ & -346.8 \\
& $-2,697.4$ & $-1,828.0$ & $-1,039.4$ & $-2,719.8$ & -2233.3 \\
Official (net) & & & & 4,7 & \\
Credit & $2,500.7$ & $4,158.9$ & $4,776.2$ & $4,696.8$ & 4090.3 \\
$\quad$ Foreign exchange & $2,584.0$ & $4,209.7$ & $4,877.8$ & $4,803.9$ & 4271.1 \\
$\quad$ Food & $1,947.1$ & $2,138.6$ & $3,104.2$ & $3,388.1$ & 3379.2 \\
$\quad$ Other & 391.9 & 925.4 & 867.0 & 590.5 & 177.3 \\
$\quad$ Debit & 245.0 & $1,145.7$ & 906.6 & 825.4 & 714.7 \\
& -83.3 & -50.8 & -101.6 & -107.1 & -180.8 \\
\hline
\end{tabular}

Source: Bank of Cape Verde.

${ }^{1}$ Includes unclassified food and equipment aid. 
Table 24. Cape Verde: Government External Debt, 2001-2005

(Millions of U.S. dollars, unless otherwise indicated)

\begin{tabular}{|c|c|c|c|c|c|}
\hline & 2001 & 2002 & 2003 & 2004 & 2005 \\
\hline Total & 298.7 & 383.0 & 506.5 & 548.0 & 503.9 \\
\hline Multilaterals & 222.9 & 292.0 & 381.1 & 426.7 & 395.6 \\
\hline IMF & 0.0 & 3.3 & 7.6 & 9.6 & 12.7 \\
\hline IDA & 100.3 & 142.8 & 194.1 & 222.5 & 213.9 \\
\hline Others & 122.6 & 145.9 & 179.4 & 194.6 & 169.0 \\
\hline Official Bilaterals & 68.2 & 76.6 & 104.6 & 101.5 & 93.9 \\
\hline Paris Club & 40.3 & 46.8 & 72.1 & 68.8 & 65.3 \\
\hline Non-Paris Club & 17.0 & 18.7 & 22.3 & 24.9 & 23.8 \\
\hline Commercial & 7.6 & 14.3 & 20.7 & 19.7 & 14.4 \\
\hline Multilateral & 222.9 & 292.0 & 381.1 & 426.7 & 395.6 \\
\hline AfDF $^{1}$ & 77.5 & 94.9 & 118.6 & 129.9 & 114.7 \\
\hline IDA & 100.3 & 142.8 & 194.1 & 222.5 & 213.9 \\
\hline IMF & 0.0 & 3.3 & 7.6 & 9.6 & 12.7 \\
\hline BADEA $^{1}$ & 16.0 & 19.8 & 25.0 & 28.3 & 23.9 \\
\hline $\mathrm{AfDB}^{1}$ & 0.2 & 0.1 & 0.0 & 0.0 & 0.0 \\
\hline $\mathrm{EIB}^{1}$ & 7.6 & 6.8 & 6.9 & 5.9 & 4.1 \\
\hline OPEC $^{1}$ & 6.0 & 5.7 & 6.7 & 6.5 & 5.8 \\
\hline $\mathrm{IFAD}^{1}$ & 7.7 & 10.0 & 12.5 & 14.1 & 12.4 \\
\hline Saudi Fund ${ }^{1}$ & 0.4 & 0.3 & 0.0 & 0.0 & 0.0 \\
\hline $\mathrm{NDF}^{1}$ & 2.2 & 2.6 & 3.3 & 3.5 & 3.0 \\
\hline NTF $^{1}$ & 5.0 & 5.6 & 6.4 & 6.4 & 5.2 \\
\hline Bilateral & 74.6 & 84.0 & 113.5 & 109.7 & 99.6 \\
\hline Government & 57.4 & 65.6 & 94.5 & 93.7 & 89.1 \\
\hline China & 12.7 & 15.1 & 18.2 & 19.7 & 17.0 \\
\hline Kuwait & 4.3 & 3.6 & 4.1 & 5.2 & 6.8 \\
\hline Portugal & 24.5 & 29.2 & 34.4 & 32.2 & 33.8 \\
\hline South Africa & $\ldots$ & $\ldots$ & $\ldots$ & $\ldots$ & $\ldots$ \\
\hline Abu Dhabi & 0.1 & 0.0 & 0.0 & 0.0 & 0.0 \\
\hline Germany & 9.5 & 10.3 & 11.1 & 10.6 & 8.0 \\
\hline Russia & 0.0 & 0.0 & 17.8 & 17.8 & 17.8 \\
\hline Export credit agencies & 17.1 & 18.4 & 19.0 & 16.0 & 10.5 \\
\hline Caisse Général des Dépôts & 4.2 & 5.1 & 4.2 & 2.7 & 0.9 \\
\hline ICO (Spain) & 6.2 & 7.3 & 8.8 & 8.2 & 5.7 \\
\hline CACEX (Brazil) & 2.3 & 2.7 & 3.3 & 3.6 & 3.1 \\
\hline SOMEC (Portugal) & 4.4 & 3.2 & 2.7 & 1.6 & 0.8 \\
\hline Private companies & 7.6 & 14.3 & 20.7 & 19.7 & 14.4 \\
\hline Banco Espirito Santo & 7.6 & 14.3 & 20.7 & 19.7 & 14.4 \\
\hline MSF (Portugal) & $\ldots$ & $\ldots$ & $\ldots$ & $\ldots$ & $\ldots$ \\
\hline Total (excluding arrears) & 305.1 & 390.3 & 515.3 & 556.1 & 509.6 \\
\hline Stock of external debt arrears & 14.5 & 7.5 & 17.2 & 0.0 & 0.0 \\
\hline Total (including arrears) & 319.5 & 397.9 & 532.5 & 556.1 & 509.6 \\
\hline
\end{tabular}

Source: Ministry of Finance and Public Administration.

${ }^{1}$ AfDF = African Development Fund; BADEA = Arab Bank for Economic Development for Africa; AfDB = African Development Bank; EIB = European Investment Bank;

OPEC = Organization of Petroleum Exporting Countries; IFAD = International Fund for Agricultural Development;

Saudi Fund = Saudi Fund for Development; NDF = Nordic Development Fund; and NTF = Nigeria Trust Fund. 


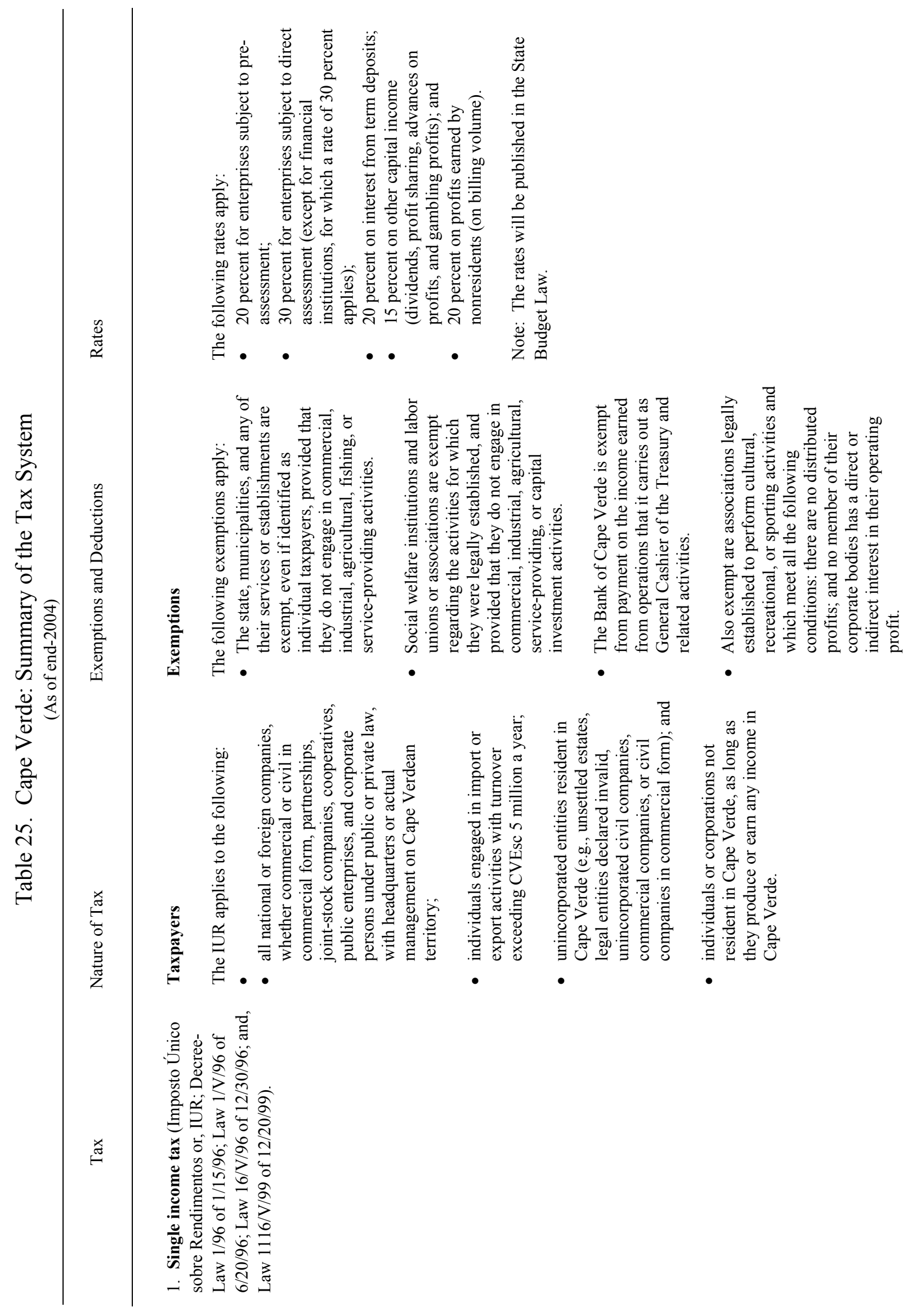




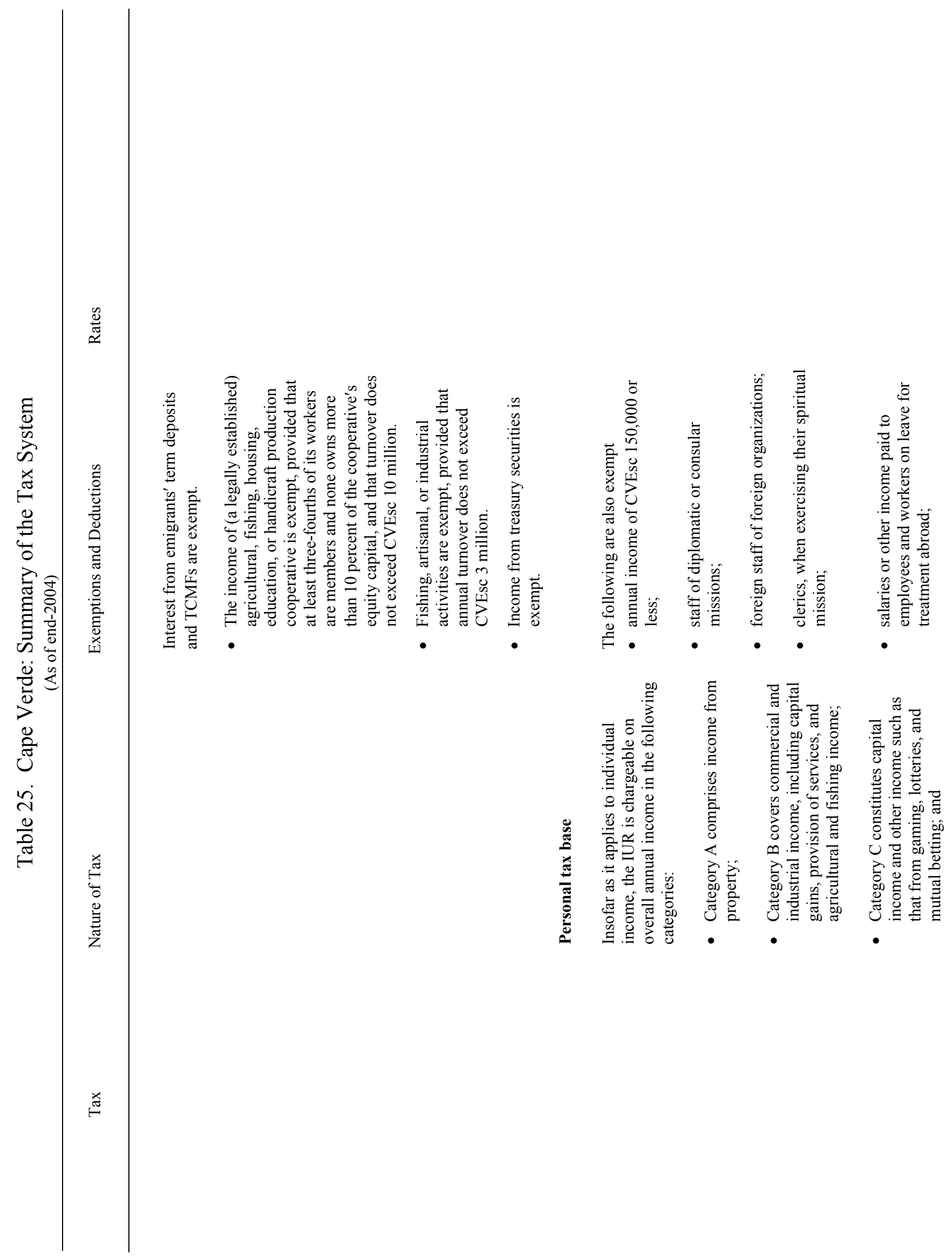




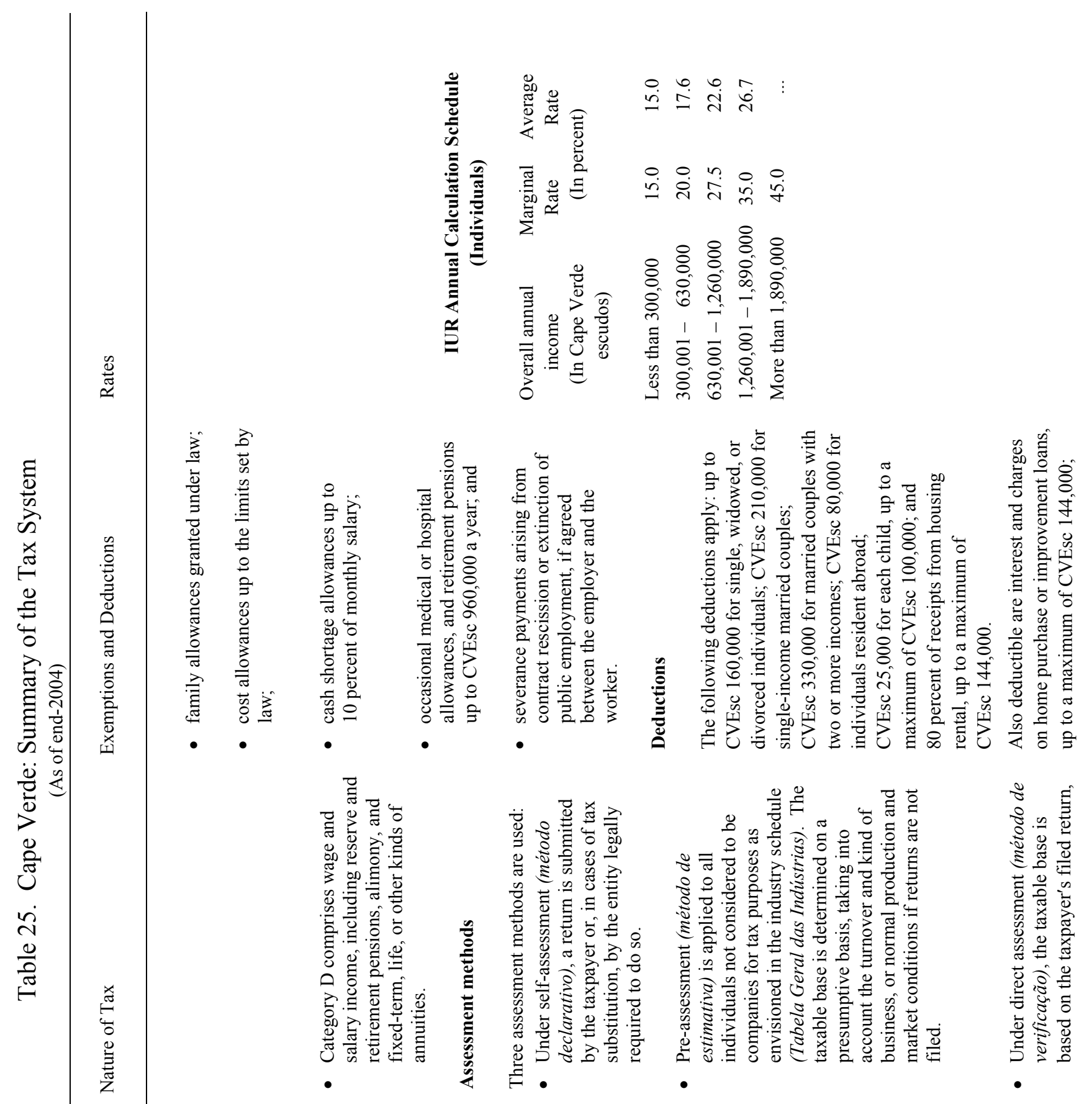




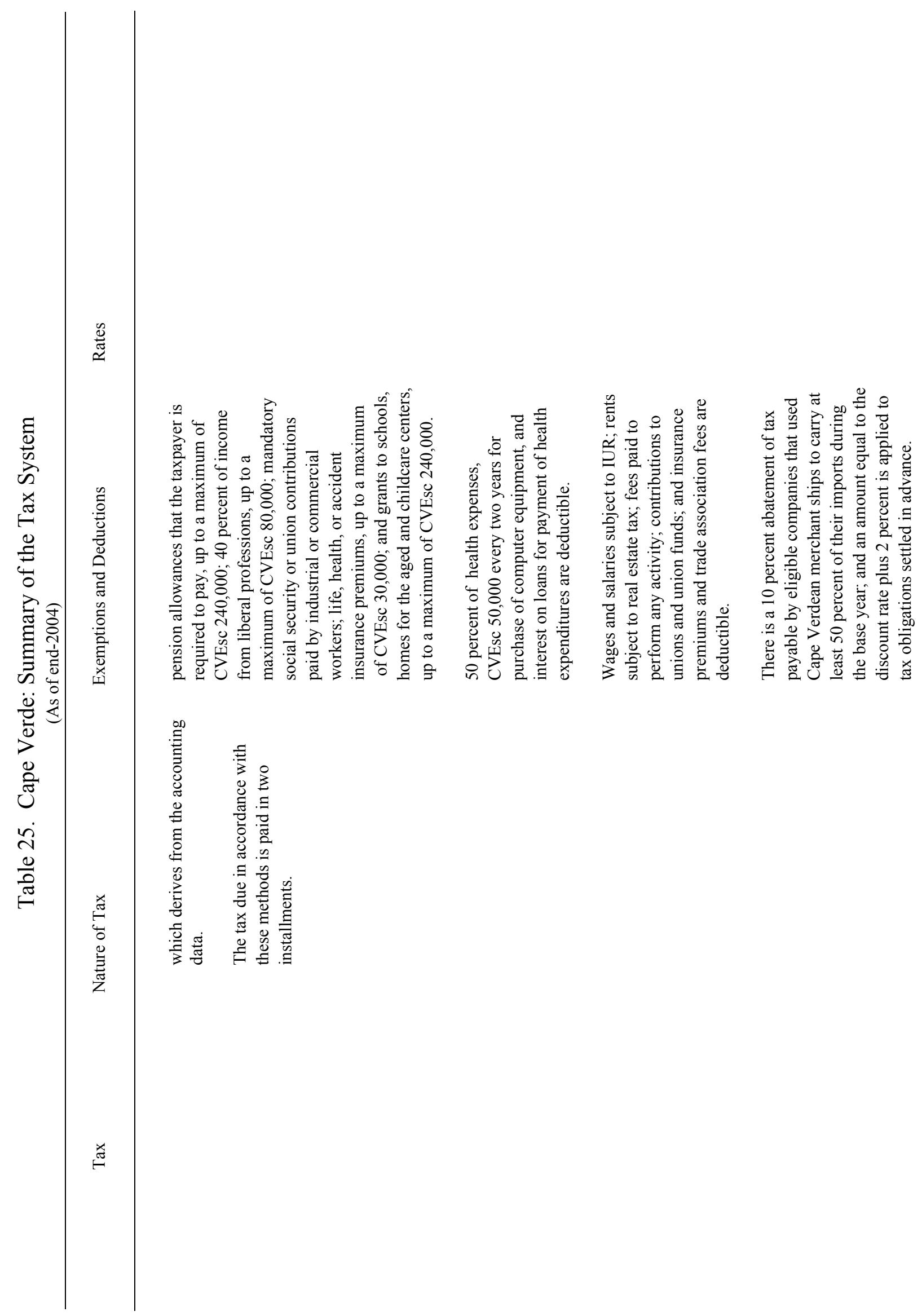




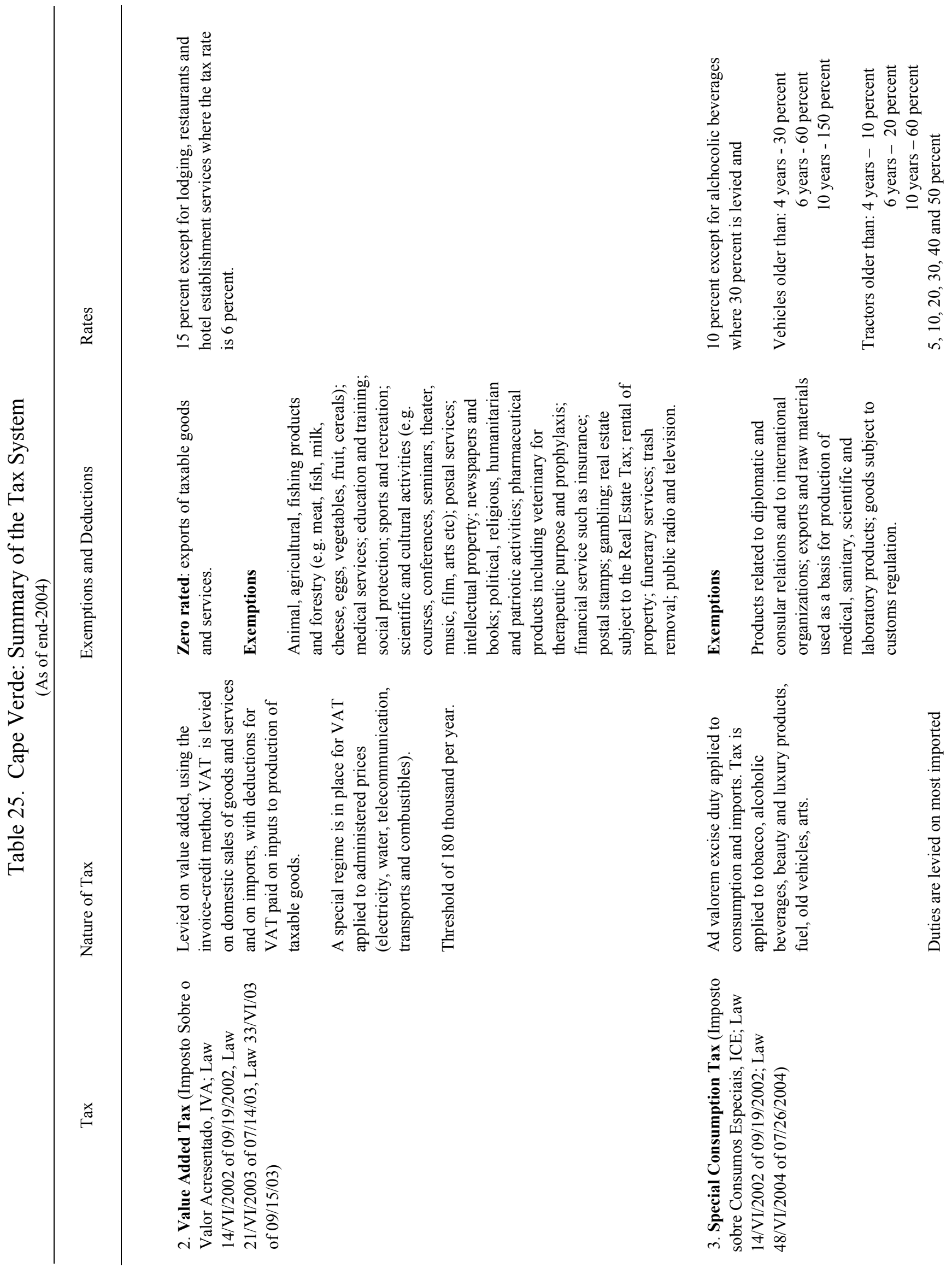




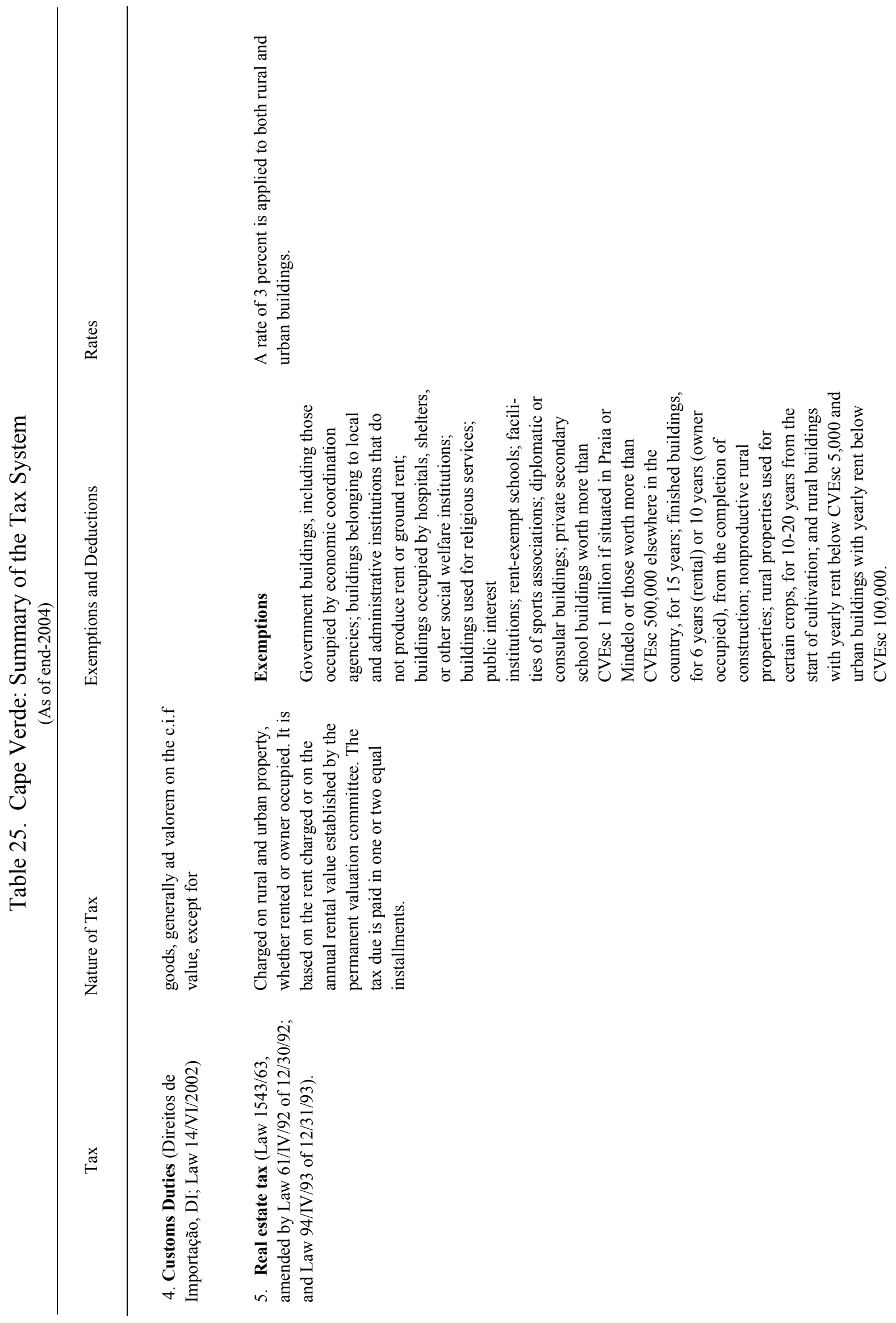




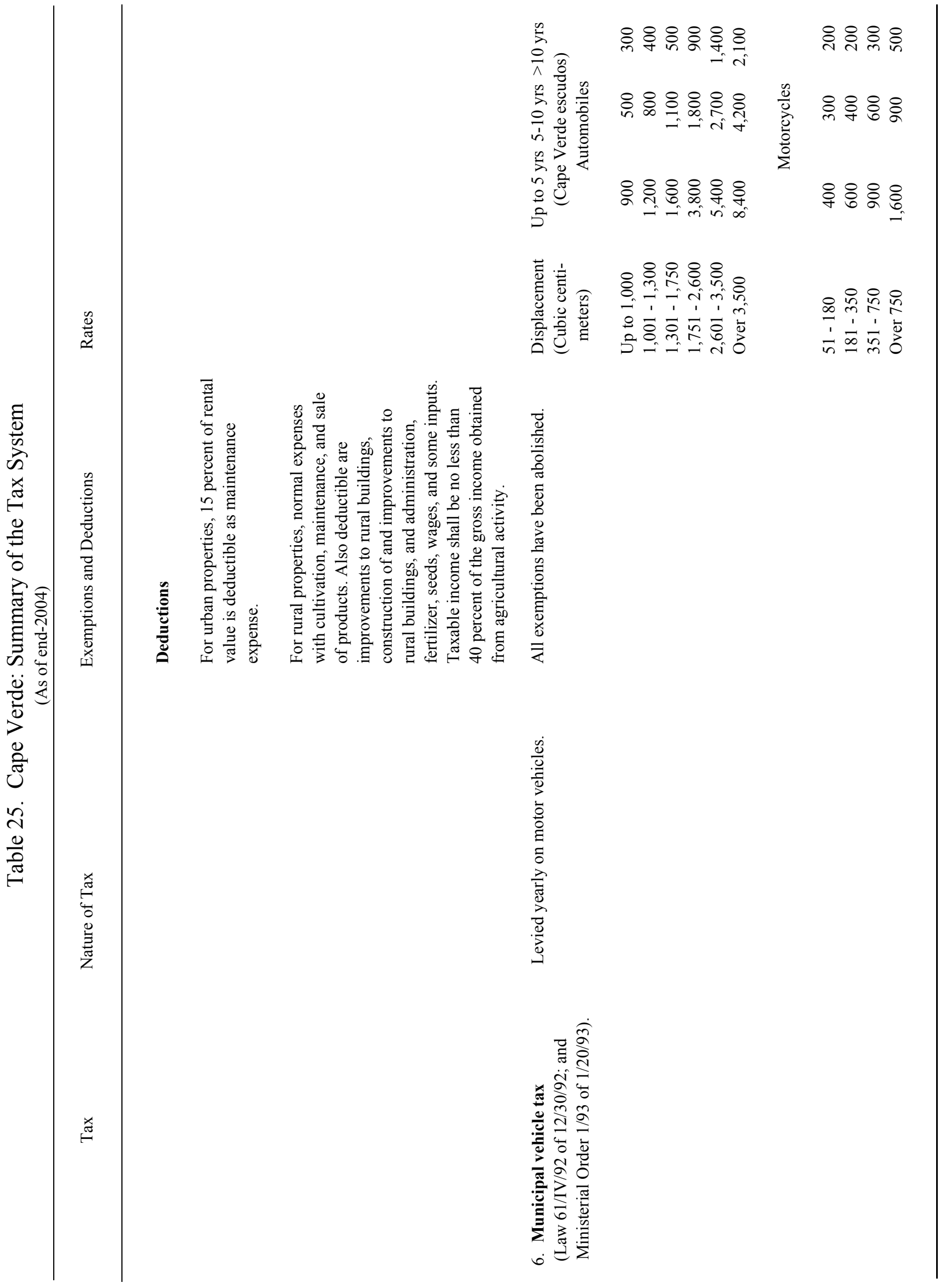

